\title{
On the Role of Clustering in Personalized PageRank Estimation
}

\author{
DANIEL VIAL and VIJAY SUBRAMANIAN, University of Michigan
}

Personalized PageRank (PPR) is a measure of the importance of a node from the perspective of another (we call these nodes the target and the source, respectively). PPR has been used in many applications, such as offering a Twitter user (the source) recommendations of whom to follow (targets deemed important by PPR); additionally, PPR has been used in graph-theoretic problems such as community detection. However, computing PPR is infeasible for large networks like Twitter, so efficient estimation algorithms are necessary.

In this work, we analyze the relationship between PPR estimation complexity and clustering. First, we devise algorithms to estimate PPR for many source/target pairs. In particular, we propose an enhanced version of the existing single pair estimator Bidirectional-PPR that is more useful as a primitive for many pair estimation. We then show that the common underlying graph can be leveraged to efficiently and jointly estimate PPR for many pairs rather than treating each pair separately using the primitive algorithm. Next, we show the complexity of our joint estimation scheme relates closely to the degree of clustering among the sources and targets at hand, indicating that estimating PPR for many pairs is easier when clustering occurs. Finally, we consider estimating PPR when several machines are available for parallel computation, devising a method that leverages our clustering findings, specifically the quantities computed in situ, to assign tasks to machines in a manner that reduces computation time. This demonstrates that the relationship between complexity and clustering has important consequences in a practical distributed setting.

CCS Concepts: • Mathematics of computing $\rightarrow$ Graph algorithms; • Theory of computation $\rightarrow$ Distributed algorithms; Random walks and Markov chains;

Additional Key Words and Phrases: Personalized PageRank, clustering, social and information networks

\section{ACM Reference format:}

Daniel Vial and Vijay Subramanian. 2019. On the Role of Clustering in Personalized PageRank Estimation. ACM Trans. Model. Perform. Eval. Comput. Syst. 4, 4, Article 21 (December 2019), 33 pages.

https://doi.org/10.1145/3366635

\section{INTRODUCTION}

Many systems, ranging from social networks to financial markets to the human brain, can be represented as graphs. When analyzing such systems, questions regarding the importance of nodes and the relationships between them arise. Which nodes are most influential, globally and locally? From the perspective of a given node, which nodes are important, and which nodes consider the given node to be important? How can these notions be quantified?

We are grateful for financial support from the NSF (grant numbers AST-1343381, AST-1516075, IIS-1538827 and ECCS1608361).

Authors' address: D. Vial, 4303 EECS, 1301 Beal Ave, Ann Arbor, MI 48109; email: dvial@umich.edu; V. Subramanian, 4112 EECS, 1301 Beal Ave, Ann Arbor, MI 48109; email: vgsubram@umich.edu.

Permission to make digital or hard copies of all or part of this work for personal or classroom use is granted without fee provided that copies are not made or distributed for profit or commercial advantage and that copies bear this notice and the full citation on the first page. Copyrights for components of this work owned by others than ACM must be honored. Abstracting with credit is permitted. To copy otherwise, or republish, to post on servers or to redistribute to lists, requires prior specific permission and/or a fee. Request permissions from permissions@acm.org.

(C) 2019 Association for Computing Machinery.

2376-3639/2019/12-ART21 \$15.00

https://doi.org/10.1145/3366635 
PageRank and Personalized PageRank (PPR) can help answer such questions. PageRank is a measure of the importance or centrality of a target node; PPR "personalizes" this measure to the perspective of a source node. Proposed to rank Internet search results [24], and later to personalize these rankings [13], PageRank and PPR have been used in applications such as recommending Twitter followers [12] and YouTube videos [6], as well as "beyond the Web" [11], in fields such as bioinformatics $[10,23]$. In graph theory, PPR has been used as a primitive for tasks such as detecting communities near a seed node [2] and assessing similarity between graphs [15].

The widespread use of PageRank and PPR can be attributed to the notion of relational "importance" they convey, as well as the simplicity of the model from which they are derived. However, the scale of modern networks often makes them difficult (or impossible) to compute. As such, strategies for efficient estimation of PageRank and PPR are necessary.

Our contributions. In this work, we analyze the relationship between clustering and PPR estimation complexity. In particular, we devise algorithms to estimate PPR for many source/target pairs, and we show that the complexity of these methods decreases with increased clustering among the sources and targets at hand. To demonstrate the consequences of our findings, we consider a distributed setting in which this relationship between complexity and clustering can be leveraged to design more efficient algorithms. More specifically, our contributions are as follows:

(1) In Section 4, we propose an enhancement of Bidirectional-PPR [20], the state-of-the-art PPR estimator for a single source/target pair. As the name suggests, Bidirectional-PPR estimates PPR in two stages: random walks forward from the source node and dynamic programming (DP) backward from the target node. Our algorithm, called FW-BW-MCMC, adds a DP stage forward from the source that allows it to serve as a primitive in the many pair setting. In Appendix A, we establish similar guarantees to those for Bidirectional-PPR.

(2) In Section 5, we use FW-BW-MCMC as a primitive to estimate PPR for many pairs, proposing methods that accelerate the naive scheme of separately sampling random walks for each source and separately running DP for each target. For the sources, we show that forward DP allows random walk samples to be shared, decreasing the number of samples required. For the targets, we define a new iterative update for the backward DP, which eliminates repeated computations that may occur when treating each target separately. Using these ideas, we devise an algorithm with accuracy guarantees on each scalar estimate and two algorithms with accuracy guarantees on the matrix containing all estimates. Across a diverse set of graphs, our methods are roughly 1.1 to 9.3 times faster than baseline methods (Figure 1(a)).

(3) We show analytically in Section 5 and empirically in Section 6 that the accelerations offered by our algorithms are most significant when the sources and targets are each clustered together in the graph (i.e., PPR estimation is "easier" when clustering occurs). For example, our algorithms typically accelerate baseline methods by factors of 3 to 4 when clustering occurs (Figure 1(a)). More specifically, we prove the number of random walks for the sources and the number of DP iterations for the targets scale with quantities that describe clustering among the sources and targets, respectively; we find empirically that these clustering quantities scale with a more traditional clustering quantity, conductance (Figure 1(b)). In addition, although these clustering quantities are difficult to analyze in general, we provide analytical results for the stochastic block model, the prototypical model for networks with community structure.

(4) Finally, in Section 7, we demonstrate an application of our results, showing that our findings can be used to devise efficient algorithms when estimating PPR in a distributed setting. Specifically, we show that quantities computed during the forward DP can be used 


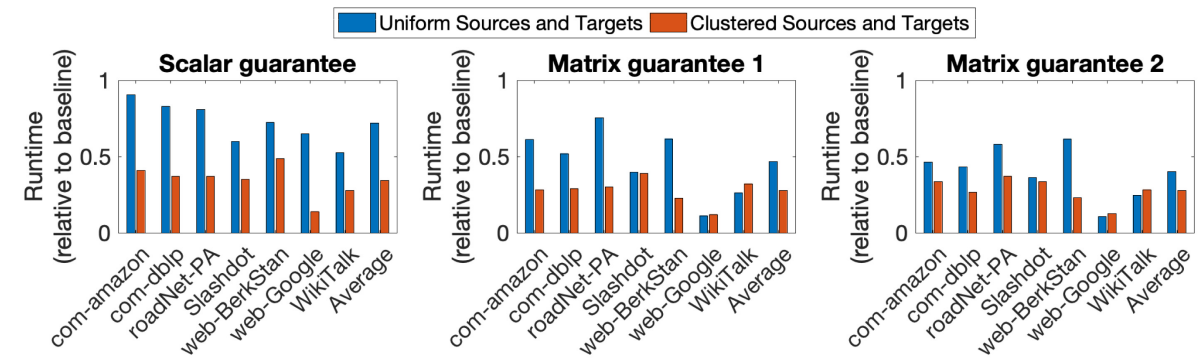

(a) Across a diverse set of real graphs, our algorithms accelerate baseline methods; these accelerations are most significant when the sources and targets are clustered (experiment details in Section 6.2).

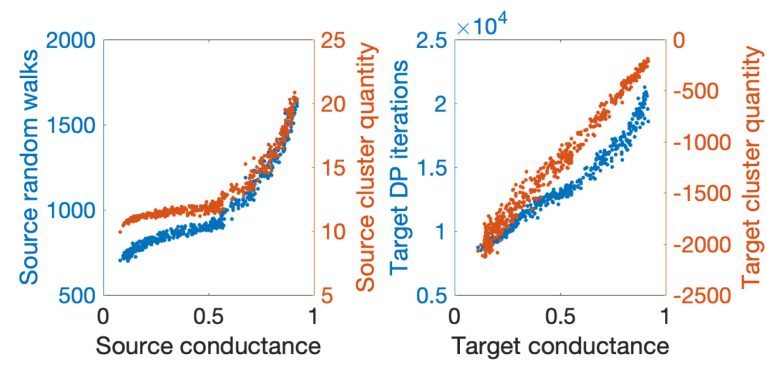

(b) The complexity of the source and target stages of our methods scale with quantities that describe clustering of sources and targets, and that behave like conductance (experiment details in Section 6.1.1).

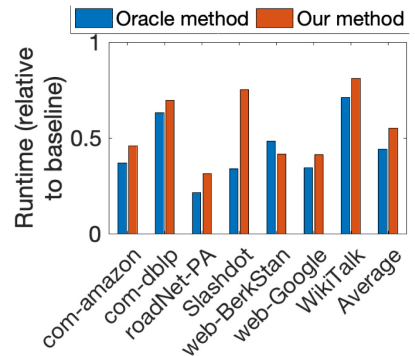

(c) Our findings can be used to identify clustering at runtime and accelerate PPR estimation (see Section 7).

Fig. 1. Key empirical results.

to predict the random walk sampling time for different assignments of tasks to machines, and we propose a natural but heuristic method to compute an assignment that (locally) minimizes this time. At a high level, our method "learns" the clustering structure present at runtime; empirically, this learning is quite successful, in the sense that our method performs nearly as well as an oracle method that knows the clustering structure a priori (Figure 1(c)).

The remainder of the article is organized as follows. We begin with preliminaries and related work in Sections 2 and 3, respectively. Sections 4 through 7 follow the preceding outline. We close in Section 8 . We also note that Table 1 contains a summary of notation used throughout the article.

\section{PRELIMINARIES}

We begin with some preliminary definitions. Let $G=(V, E)$ be a directed graph, and define $n=|V|, m=|E|$. For $v \in V$, let $N_{\text {out }}(v)=\{u \in V: v \rightarrow u \in E\}$ denote $v$ 's outgoing neighbors, and let $d_{\text {out }}(v)=\left|N_{\text {out }}(v)\right|$ denote the out-degree of $v$. For simplicity, we assume $d_{\text {out }}(v)>0 \forall v \in V$. Similarly define $N_{\text {in }}(v)$ and $d_{\text {in }}(v)$ as $v$ 's incoming neighbors and in-degree. Finally, let $A$ denote the adjacency matrix of $G$, let $D$ be the diagonal matrix with $D(v, v)=d_{\text {out }}(v)$, and define $P=D^{-1} A$.

PageRank [24] is the stationary distribution $\{\pi(v)\}_{v \in V}$ of the Markov chain with transition matrix $(1-\alpha) P+\alpha \frac{1}{n} 1_{n} 1_{n}^{T}$, where $\alpha \in(0,1)$ and $1_{n}$ denotes the all ones vector of length $n$. In words, this chain is a random walk on $G$ for which, with probability $\alpha$ at each step, the random walker "jumps" to a uniform node rather than following the walk. Let us denote this chain by $\left\{X_{i}\right\}_{i \in \mathbb{N}}$. Clearly, $\left\{X_{i}\right\}_{i \in \mathbb{N}}$ is irreducible and aperiodic, as $P(u, v) \geq \frac{\alpha}{n}>0 \forall u, v \in V$. Assuming $V$ is finite, positive recurrence follows, so $\{\pi(v)\}_{v \in V}$ exists, is unique, and satisfies 
Table 1. Summary of Notation

\begin{tabular}{|c|c|c|}
\hline Notation & Defined in & Description \\
\hline$\pi, \pi_{s}$ & Section 2 & Global PageRank vector, PPR vector for $s \in V$ \\
\hline$p^{s}, r^{s}$ & Section 4 & Output vectors of Algorithm 1 (forward DP); satisfy (5) \\
\hline$\sigma_{s}$ & Section 4 & Starting distribution for walks in FW-BW-MCMC; $\sigma_{s}=r^{s} /\left\|r^{s}\right\|_{1}$ \\
\hline$r_{\max }^{s}$ & Section 4 & Upper bound on $\left\|r^{s}\right\|_{1}$ at conclusion of Algorithm 1 \\
\hline$p^{t}, r^{t}$ & Section 4 & Output vectors of Algorithm 2 (backward DP); satisfy (6) \\
\hline$r_{\max }^{t}$ & Section 4 & Upper bound on $\left\|r^{t}\right\|_{\infty}$ at conclusion of Algorithm 2 \\
\hline$\Sigma_{S}$ & Section 5.1 .1 & Matrix with rows $\left\{\sigma_{s}\right\}_{s \in S}$ (subscript $S$ omitted at times) \\
\hline$\left\|\Sigma_{S}\right\|_{\infty, 1}$ & Section 5.1 .1 & Source clustering quantity; see (11) \\
\hline$c_{T}$ & Section 5.1 .2 & Target clustering quantity; see (16) \\
\hline $\operatorname{srank}(A)$ & Section 5.2 & Stable rank of matrix $A ; \operatorname{srank}(A)=\left(\|A\|_{F} /\|A\|_{2}\right)^{2}$ \\
\hline$P_{S}, R_{S}, P_{T}, R_{T}$ & Section 5.2 & Matrices containing $\left\{p^{s}\right\}_{s \in S},\left\{r^{s}\right\}_{s \in S},\left\{p^{t}\right\}_{t \in T},\left\{r^{t}\right\}_{t \in T}$, respectively \\
\hline$\Pi(S, T)$ & Section 5.2 & Matrix containing $\left\{\pi_{s}(t)\right\}_{s \in S, t \in T}$ \\
\hline$\sigma_{\mathrm{avg}}, \sigma_{\max }$ & Section 5.2 & Starting distributions for walks in matrix estimators; see (21) \\
\hline$\Phi(U)$ & Section 6.1 .1 & Conductance of $U \subset V$; see (22) \\
\hline$\sigma_{S}$ & Section 7 & Vector with $\sigma_{s}(v)=\max _{s \in S} \sigma_{s}(v)$ \\
\hline
\end{tabular}

$$
\pi(v)=\lim _{N \rightarrow \infty} \frac{1}{N} \sum_{i=0}^{N-1} \mathbb{1}_{\left\{X_{i}=v\right\}} \forall v \in V .
$$

It is by (1) that PageRank gives a measure of "importance": we consider $v$ important when $\pi(v)$ is large, which occurs when $v$ is visited often on the chain $\left\{X_{i}\right\}_{i \in \mathbb{N}}$.

We will henceforth refer to PageRank as global PageRank to distinguish it from the generalization to PPR. Formally, PPR is the stationary distribution $\left\{\pi_{\sigma}(v)\right\}_{v \in V}$ of the Markov chain with transition matrix $P_{\sigma}=(1-\alpha) P+\alpha 1_{n} \sigma^{T}$. Here, $\sigma$ is a nonnegative vector that sums to 1 ; hence, it yields a distribution on the jump locations, generalizing the uniform jumps of global PageRank. This gives $\pi_{\sigma}$ an interpretation like (1) while also accounting for the preference on jump locations given by $\sigma$.

There are two important mathematical viewpoints of PPR that serve as the foundation for many estimation techniques; we will make use of both in the sections that follow. The first viewpoint is linear algebraic. Here, we let $\pi_{\sigma}$ denote the stationary distribution as a row vector. By global balance, $\pi_{\sigma}$ satisfies $\pi_{\sigma}=\pi_{\sigma} P_{\sigma}$; solving for $\pi_{\sigma}$ (and assuming $\pi_{\sigma}$ is normalized to sum to 1 ) gives

$$
\pi_{\sigma}=\alpha \sigma^{T}(I-(1-\alpha) P)^{-1}=\alpha \sigma^{T} \sum_{i=0}^{\infty}(1-\alpha)^{i} P^{i} .
$$

Note this immediately suggests estimating PPR by computing the first $k$ terms of the summation. The second PPR viewpoint is probabilistic. Denoting by $\left\{Y_{i}\right\}_{i \in \mathbb{N}}$ the Markov chain with transition matrix $P$, and letting $L \sim$ geometric $(\alpha)$, Athreya and Stenflo [4] show

$$
\pi_{\sigma}(v)=\mathbb{P}\left[Y_{L}=v \mid Y_{0} \sim \sigma\right] \forall v \in V,
$$

which again suggests estimation techniques; in this case, using Markov chain Monte Carlo (MCMC). We note this viewpoint is closely related to exact sampling [25] and Doeblin chains $[4,22]$.

Often, we let $\sigma=e_{s}$ for some $s \in V$, where $e_{s} \in\{0,1\}^{n}$ satisfies $e_{s}(v)=\mathbb{1}_{\{v=s\}}$. In such a case, we denote PPR as $\left\{\pi_{s}(v)\right\}_{v \in V}$ and the transition matrix as $P_{s}$. In fact, using (2), one can show

$$
\pi_{\sigma}(v)=\sum_{s \in V} \sigma(s) \pi_{s}(v) \forall v \in V
$$

ACM Trans. Model. Perform. Eval. Comput. Syst., Vol. 4, No. 4, Article 21. Publication date: December 2019. 
Due to (4), many PPR estimation algorithms focus on estimating $\pi_{s}(v)$, from which extensions to estimating $\pi_{\sigma}(v)$ naturally follow; we focus on this as well throughout the article.

Finally, we argue existence and uniqueness of PPR is not a concern. Indeed, the PPR Markov chain is aperiodic (since $P_{s}(s, s) \geq \alpha>0$ ), so to guarantee existence and uniqueness, we need only verify irreducibility. For this, let $V_{s}=\{v \in V: \exists a$ path from $s$ to $v$ in $G\}$ so that $\forall u, v \in V_{s}, \exists i \in \mathbb{N}$ subject to $P_{s}^{i}(u, v)>0$ (we can jump from $u$ to $s$, then reach $v$ from $s$ ). Hence, if $P_{s}$ is not irreducible, we can define a modified chain with states $V_{s}$ that is irreducible and obtain the stationary distribution $\pi_{s}$ for this chain. We can then set $\pi_{s}(v)=0 \forall v \notin V_{s}$-intuitively, if $s$ cannot reach $v, v$ is not "important" to $s$, so its PPR should be zero. Given this simple fix, we assume existence and uniqueness of PPR.

\section{RELATED WORK}

Before proceeding, we discuss some existing PPR estimation algorithms. Broadly speaking, these can be organized hierarchically: first, those that estimate the entire PPR matrix $\left\{\pi_{s}(t)\right\}_{s \in V, t \in V \text {; }}$ second, those that estimate a single row $\left\{\pi_{s}(t)\right\}_{t \in V}$ or column $\left\{\pi_{s}(t)\right\}_{s \in V}$ of this matrix, or its column sums (i.e., global PageRank); and third, those that estimate a single entry $\pi_{s}(t)$.

At the first level, several algorithms have been proposed to accelerate the power iteration or matrix inversion in (2). To accelerate the power iteration, Jeh and Widom [14] provides a decomposition that allows a single row of the PPR matrix to be estimated using previously computed rows; hence, this yields a procedure of first computing a small number of rows and then using these to estimate other rows. To obtain less costly matrix inversions, several works (e.g., $[26,27])$ have leveraged structural assumptions of the graph at hand. For example, Tong et al. [27] propose a decomposition of $P$ into a block diagonal matrix $P_{1}$ and $P_{2}:=P-P_{1}$; for graphs like social networks, $P_{2}$ can be extremely sparse. From the probabilistic viewpoint (3), Fogaras et al. [9] gives an algorithm to estimate any entry of the PPR matrix at runtime using a precomputed database of random walk samples.

At the second level, algorithms include the DP methods in Andersen et al. [2] and Andersen et al. [1] that estimate a row and a column of the PPR matrix, respectively; both can be viewed as localized versions of the power iteration in (2). The algorithm in Andersen et al. [2] yields $l_{1}$ and $l_{\infty}$ error guarantees on the row estimate with complexity $O(m)$, whereas Andersen et al. [1] gives an $l_{\infty}$ guarantee on the column estimate with complexity $O(m)$. We make use of these algorithms in our methods and will discuss them in more detail in Section 4. We also note the approach in Andersen et al. [1,2] is closely related to work by Lee et al. [16-18] that focuses on estimation of the stationary distribution of countable state-space Markov chains, as well as estimation in the context of general linear systems. From the probabilistic viewpoint, an important work is Avrachenkov et al. [5], which analyzes Monte Carlo methods for global PageRank estimation, based on both the final step of sampled random walks (as given by (3)) and the number of visits along the entire walk. In Avrachenkov et al. [5], it is shown that a single walk from each node (i.e., $n$ walks total) suffices to obtain estimates with small relative error for nodes with high global PageRank. Another work in this category is Borgs et al. [7], which uses random walk-based methods to detect all nodes with global PageRank exceeding $n^{-\delta}, \delta \in(0,1)$ with complexity sublinear in $n$. Borgs et al. [7] also contains an algorithm to estimate a row of the PPR matrix with each estimate satisfying a multiplicative plus additive error guarantee; the complexity is linear in $n$ (if the error tolerance is set to match the guarantee used in [20]).

At the third level, the aforementioned Bidirectional-PPR algorithm from Lofgren et al. [20] combines existing DP and Monte Carlo methods to estimate a single PPR value with worst-case and average-case complexity $O(n)$ and $O(\sqrt{m})$, respectively. From an accuracy perspective, this algorithm achieves a relative error bound for PPR values exceeding $1 / n$ and an absolute error bound otherwise. We discuss this algorithm in more detail in Section 4. 


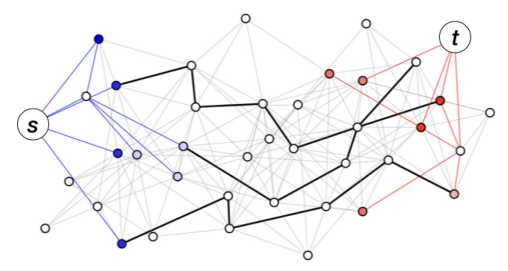

Fig. 2. Depiction of our algorithm FW-BW-MCMC. Blue and red nodes and edges show forward and backward DP, respectively; thick black edges show random walks.

In the context of this body of work, we will consider estimation of a small set of PPR values, $\left\{\pi_{s}(t)\right\}_{s \in S, t \in T}$ for some $S, T \subset V$. Although we do not precisely quantify "small," we implicitly assume $|S| \approx|T|=o(\sqrt{m})$. In this setting, the existing methods described previously can be applied in two ways. First, using methods such as the power iteration or the DP schemes (i.e., the first two levels of the preceding hierarchy), one can estimate entire rows and/or columns of the PPR matrix and then discard unwanted estimates. Such approaches typically have complexity $O(|S| m)$ or $O(|T| m)$ (depending on exactly which approach is used). Second, one can run the single pair estimator Bidirectional-PPR separately for each pair $(s, t) \in S \times T$. This approach has typical complexity $O(|S||T| \sqrt{m})$. When $|S| \approx|T|=o(\sqrt{m})$, the second approach is more efficient. Hence, we will treat this approach as a baseline for comparison to our methods. Our primary contribution is to show that this baseline can be accelerated by exploiting clustering among $S$ and $T$ to estimate PPR values jointly rather than running Bidirectional-PPR separately for each $(s, t) \in S \times T$.

\section{SINGLE NODE PAIR ESTIMATION}

We begin by proposing an enhancement of Bidirectional-PPR [20], the state-of-the-art single pair PPR estimator; we will introduce our algorithm and then describe Bidirectional-PPR as a special case. As mentioned in Section 3, the idea behind these estimators is to combine DP and MCMC to estimate $\pi_{s}(t)$ for some $s, t \in V$. Our algorithm uses two DP stages and one MCMC stage. We will refer to these stages as the forward DP, backward DP, and MCMC stages; hence, we call our estimator FW-BW-MCMC. It is depicted pictorially in Figure 2 and defined formally later in Algorithm 3. Before proceeding, we briefly describe each stage.
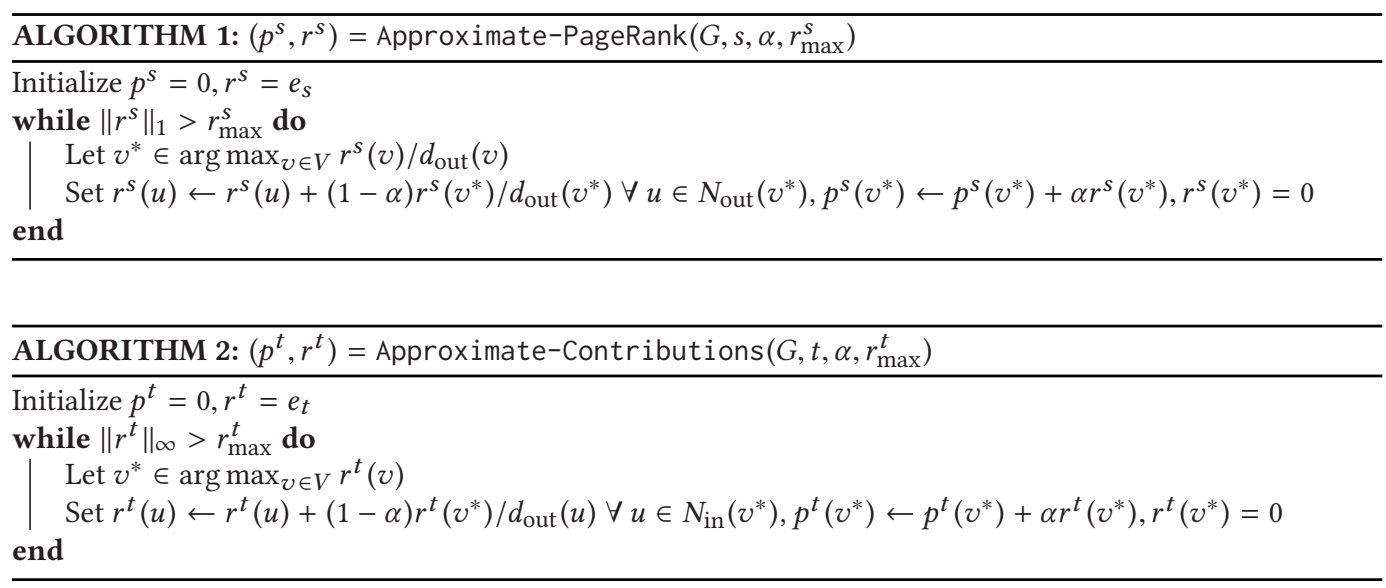

The forward DP stage is Algorithm 1. This is nearly identical to the Approximate-PageRank algorithm of Andersen et al. [2], so we use the same name here; however, we change the termination criteria from $\left\|D^{-1} r^{s}\right\|_{\infty} \leq r_{\max }^{s}$ to $\left\|r^{s}\right\|_{1} \leq r_{\max }^{s}$, where $r_{\max }^{s} \in(0,1)$ is an input to the algorithm 


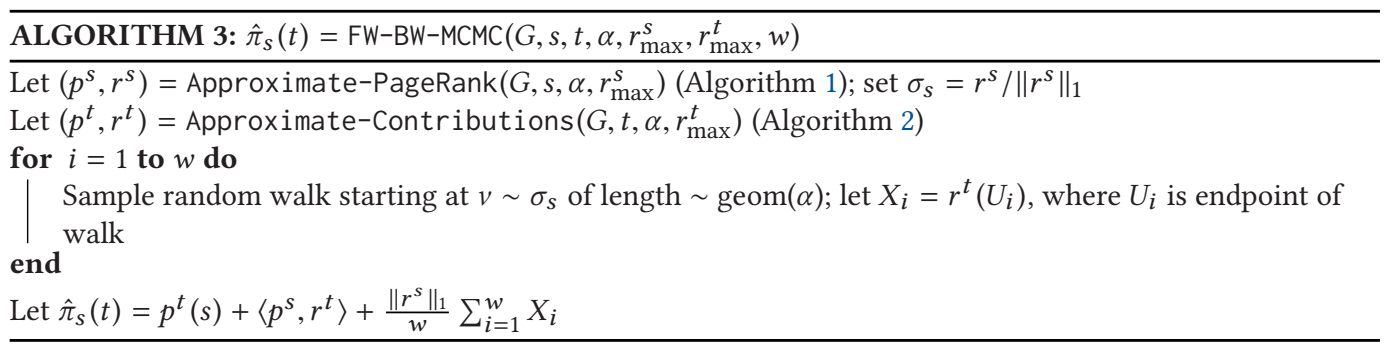

(we describe our motivation for this change shortly). The algorithm takes as input $s \in V$ and produces $p^{s}, r^{s} \in \mathbb{R}_{+}^{n}$, shown in Andersen et al. [2] to satisfy the invariant (5) at each iteration:

$$
\pi_{s}(u)=p^{s}(u)+\sum_{w \in V} r^{s}(w) \pi_{w}(u) \forall u \in V .
$$

As mentioned Section 3, Algorithm 1 can be viewed as a "localized" power iteration. At a high level, it computes elements of the matrices in (2) corresponding to high probability paths from $s$ to $u$ (the $p^{s}(u)$ term) while tracking the error from "uncomputed" paths (the $\sum_{w \in V} r^{s}(w) \pi_{w}(u)$ term). These high probability paths are shown as blue edges in Figure 2.

The backward DP stage is Approximate-Contributions (Algorithm 2, from Andersen et al. [1]), which can be viewed as the dual of Algorithm 1: although Algorithm 1 works forward (along outgoing edges), Algorithm 2 works backward (along incoming edges). In Andersen et al. [1], it is shown that Algorithm 2 maintains the invariant (6), which can be interpreted similarly to (5). This stage is shown in red in Figure 2.

$$
\pi_{v}(t)=p^{t}(v)+\sum_{w \in V} \pi_{v}(w) r^{t}(w) \forall v \in V
$$

To motivate the MCMC stage, first observe that combining (5) and (6) with $u=t$ and $v=s$ gives

$$
\pi_{s}(t)=p^{t}(s)+\left\langle p^{s}, r^{t}\right\rangle+\sum_{w, w^{\prime} \in V} r^{s}(w) \pi_{w}\left(w^{\prime}\right) r^{t}\left(w^{\prime}\right),
$$

and so, after running the DP stages, only the third term in (7) is unknown. The goal of the MCMC stage is to estimate this term. Toward this end, let $\sigma_{s}=r^{s} /\left\|r^{s}\right\|_{1}$ and use (4) to write this term as

$$
\left\|r^{s}\right\|_{1} \sum_{w^{\prime} \in V} \sum_{w \in V} \sigma_{s}(w) \pi_{w}\left(w^{\prime}\right) r^{t}\left(w^{\prime}\right)=\left\|r^{s}\right\|_{1} \sum_{w^{\prime} \in V} \pi_{\sigma_{s}}\left(w^{\prime}\right) r^{t}\left(w^{\prime}\right)=\left\|r^{s}\right\|_{1} \mathbb{E}_{U \sim \pi_{\sigma_{s}}}\left[r^{t}(U)\right] .
$$

Leveraging the probabilistic PPR interpretation (3), we can then estimate this term by sampling random walks. More specifically, we first sample a starting node from $\sigma_{s}$ (blue nodes in Figure 2), and we then sample a random walk beginning at the starting node (black edges in Figure 2). This process of sampling random walks is the MCMC stage of our algorithm.

As mentioned earlier, the forward DP stage uses termination criteria $\left\|r^{s}\right\|_{1} \leq r_{\max }^{s}$ rather than the $\left\|D^{-1} r^{s}\right\|_{\infty} \leq r_{\max }^{s}$ criteria used in Andersen et al. [2]. This is because we will require a uniform bound on $\left\{\left\|r^{s}\right\|_{1}\right\}_{s \in S}$ when proving results pertaining to a set sources $S$ in later sections. However, this bound is not needed in practice, where we can instead use $\left\|D^{-1} r^{s}\right\|_{\infty} \leq r_{\max }^{s}$ termination. We call this variant of our algorithm FW-BW-MCMC-Practical; see Algorithm 8 in Appendix D for a formal definition.

Having defined FW-BW-MCMC, we can describe the existing algorithm Bidirectional-PPR, which (in brief) operates as follows: run the backward DP from $t$, take $v=s$ in (6), and estimate the unknown term $\mathbb{E}_{U \sim \pi_{s}}\left[r^{t}(U)\right]$ by sampling random walks from $s$. We observe this is a special case of FW-BW-MCMC; specifically, the case $r_{\max }^{s}=1$. We reemphasize that walks are sampled from 
$v \sim \sigma_{s}$ in FW-BW-MCMC and from $s$ in Bidirectional-PPR, which will be an important distinction later.

In the next sections, we will propose many pair estimators that use either Bidirectional-PPR or our enhancement as a primitive. We will show that using our enhancement as the primitive offers runtime accelerations not possible when using Bidirectional-PPR. Implicit in this discussion will be an understanding that using either primitive yields similar performance when these accelerations are ignored (so that using our enhancement as the primitive offers better performance when the accelerations are accounted for). In particular, we can prove the following results (as single pair estimation is not our focus, we defer formal statements and proofs to Appendix A):

(1) FW-BW-MCMC, FW-BW-MCMC-Practical, and Bidirectional-PPR offer the same accuracy guarantee (except for mild differences in assumptions).

(2) FW-BW-MCMC and Bidirectional-PPR have worst-case complexity $O(n)$.

(3) FW-BW-MCMC-Practical and Bidirectional-PPR have average-case complexity $O(\sqrt{m})$.

\section{MANY NODE PAIR ESTIMATION}

In this section, we consider the problem of estimating PPR for many node pairs, $\left\{\pi_{s}(t)\right\}_{s \in S, t \in T}$ for some $S, T \subset V$. We consider two variants of this problem. First, in Section 5.1, we view $\left\{\pi_{s}(t)\right\}_{s \in S, t \in T}$ as a set of scalars, each of which we aim to accurately estimate. Second, in Section 5.2, we view $\left\{\pi_{s}(t)\right\}_{s \in S, t \in T}$ as a matrix, which we aim to approximate accurately in the operator norm. For both variants, we propose algorithms that accelerate existing approaches, and we show the accelerations scale with quantities that can be interpreted as clustering measures of $S$ and $T$. In addition to these algorithms, we briefly discuss variants that use precomputation in Section 5.3.

\subsection{Scalar Estimation Viewpoint}

Given $S, T \subset V$, a natural approach to estimate $\pi_{s}(t) \forall(s, t) \in S \times T$ is to use single pair estimators from Section 4 as primitives. In particular, we could use either of the following approaches:

- Run forward DP and sample random walks from $v \sim \sigma_{s}$ for each $s \in S$. Run backward DP from each $t \in T$. Compute estimates as in FW-BW-MCMC.

- Sample random walks from each $s \in S$. Run backward DP from each $t \in T$. Compute estimates as in Bidirectional-PPR.

As argued in Appendix A, the primitives FW-BW-MCMC and Bidirectional-PPR are roughly equivalent in terms of complexity and accuracy; hence, both approaches have similar complexity. However, in Section 5.1.1, we show the source stage of the first approach (forward DP and random walks) can be accelerated in a way not possible for the second approach. Further, in Section 5.1.2, we show the target stage (backward DP) can be accelerated as well. Hence, using primitive method FW-BW-MCMC and the accelerations of Sections 5.1.1 and 5.1.2, we can more efficiently estimate $\left\{\pi_{s}(t)\right\}_{s \in S, t \in T \text {. }}$

5.1.1 Source Stage Acceleration. To accelerate the source stage, we define a unified MCMC stage for a set of sources $S$. At a high level, this scheme allows us to share walks across multiple $s \in S$, thereby decreasing the total number of walks required. We motivate the scheme pictorially in Figure 3(a), for the simple case $S=\left\{s_{1}, s_{2}\right\}$. Here, blue and red depict $\sigma_{s_{1}}$ and $\sigma_{s_{2}}$ values-that is, blue and red nodes are the starting nodes of random walks used in the $\pi_{s_{1}}$ and $\pi_{s_{2}}$ estimates, respectively. Observe several nodes have nonzero $\sigma_{s_{1}}$ and $\sigma_{s_{2}}$ values. The unified MCMC stage allows us to use random walks sampled from such nodes toward both estimates $\left(\pi_{s_{1}}\right.$ and $\left.\pi_{s_{2}}\right)$. 


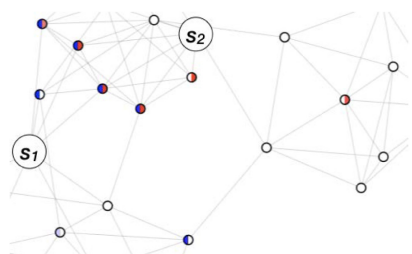

(a) Walks are sampled from blue nodes for $s_{1}$ and from red nodes for $s_{2}$; walks from blue and red nodes are shared between $s_{1}$ and $s_{2}$.

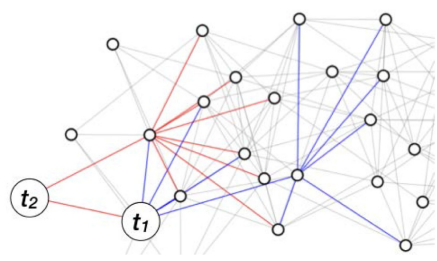

(b) Red paths are computed via Extend during $t_{2}$ DP; blue paths can be computed via Merge during $t_{2}$ DP, rather than recomputed via Extend.

Fig. 3. Accelerations for FW-BW-MCMC when $|S|=|T|=2$.

To define the unified MCMC stage, we first define an equivalent $\mathrm{MCMC}$ stage for a single source. Recall that in Algorithm 3 we sample each of $w$ random walks in two stages: first, we sample starting node $v_{s} \sim \sigma_{s}$, and second, we sample a walk starting at $v_{s}$. Equivalently, we can first sample starting nodes $\left\{v_{s}^{(i)}\right\}_{i=1}^{w}$ independent and identically distributed from $\sigma_{s}$, and then sample $X_{s}^{(w)}(v):=\sum_{i=1}^{w} \mathbb{1}_{\left\{v_{s}^{(i)}=v\right\}}$ walks starting at $v$, for each $v \in V$. With this in mind, the unified MCMC stage proceeds as follows. First, for each $s \in S$, we sample starting nodes $\left\{v_{s}^{(i)}\right\}_{i=1}^{w}$ independent and identically distributed from $\sigma_{s}$ (as in the single source case), and we define $X_{s}^{(w)}(v)$ as earlier. Next, we sample $X^{(w)}(v):=\max _{s \in S} X_{s}^{(w)}(v)$ walks starting at each $v \in V$. Letting $U_{i}^{v}$ denote the endpoint of the $i$-th walk from $v$, we then estimate $\pi_{s}(t)$ as

$$
\hat{\pi}_{s}(t)=p^{t}(s)+\left\langle p^{s}, r^{t}\right\rangle+\frac{\left\|r^{s}\right\|_{1}}{w} \sum_{v \in V: X_{s}^{(w)}(v)>0} \sum_{i=1}^{X_{s}^{(w)}(v)} r^{t}\left(U_{i}^{v}\right) .
$$

The final term in (8) is an unbiased estimate of $\mathbb{E}_{U \sim \pi_{\sigma s}}\left[r^{t}(U)\right]$ using $\sum_{v \in V} X_{s}^{(w)}(v)=w$ random walks, so the accuracy guarantee of Algorithm 3 holds. To analyze the complexity of this scheme, first note we must sample $\sum_{v \in V} X^{(w)}(v)$ walks in total. We can then prove the following.

Theorem 5.1. Fix $\epsilon, p_{\text {fail }} \in(0,1)$. Assume $w$, the number of random walks required for each $s \in S$ in the unified MCMC stage from Section 5.1.1, satisfies

$$
w>\frac{3 \log \left(2 \sum_{s \in S, v \in V} \mathbb{1}_{\left\{\sigma_{s}(v)>0\right\}} / p_{\text {fail }}\right)}{\epsilon^{2} \min _{s \in S, v \in V: \sigma_{s}(v)>0} \sigma_{s}(v)} .
$$

Then with probability at least $1-p_{\text {fail }}$, the total number of walks $\sum_{v \in V} X^{(w)}(v)$ sampled satisfies

$$
\left|\sum_{v \in V} X^{(w)}(v)-w \sum_{v \in V} \max _{s \in S} \sigma_{s}(v)\right| \leq \epsilon w \sum_{v \in V} \max _{s \in S} \sigma_{s}(v) .
$$

Proof. See Appendix E.

Before proceeding, we offer several remarks on this result:

- A lower bound on $w$ is given by (27) in Theorem A.1 to guarantee accuracy of each estimate. Thus, if $w$ exceeds both (9) and (27), we obtain guarantees for both scalar accuracy and complexity of the walks. (In general, though, it is unclear which of (9) and (27) is larger.)

- In the worst case, the denominator on the right side of (9) may be quite small, so the assumption on $w$ in Theorem 5.1 may be restrictive. However, this only means that the concentration in (10) may not provably occur, not that the scheme will necessarily have poor 
performance. Furthermore, we find that this concentration essentially occurs for the values of $w$ used in practice (e.g., later, see the leftmost plot in Figure 5 and the left two plots in Figure 8).

- We will denote the matrix with rows $\left\{\sigma_{s}\right\}_{s \in S}$ by $\Sigma$ (or by $\Sigma_{S}$, if we wish to emphasize the sources $S$ at hand) and will write the bound in (10) as

$$
\|\Sigma\|_{\infty, 1}=\sum_{v \in V} \max _{s \in S} \sigma_{s}(v) .
$$

Here, we have used the notation of the $L_{p, q}$ matrix norm, defined for a matrix $A$ as

$$
\|A\|_{p, q}=\left(\sum_{j}\left(\sum_{i}|A(i, j)|^{p}\right)^{q / p}\right)^{1 / q} .
$$

From Theorem 5.1, we expect to sample approximately $w\|\Sigma\|_{\infty, 1}$ walks when $w$ is large. It is easily verified that $\|\Sigma\|_{\infty, 1} \in[1,|S|]$, so our approach requires $w|S|$ random walks in the worst case but only $w$ in the best case. In contrast, if we use Bidirectional-PPR as a primitive for many pair estimation, the unified MCMC stage is not possible (all random walks used to estimate $\pi_{s}$ begin at $s$, so sharing walks is not possible), and $w|S|$ walks are always required. In short, FW-BW-MCMC with the unified MCMC stage accelerates the source stage of our many pair estimation approach.

Unfortunately, it is difficult to quantify the degree of this acceleration in general, in part because $\left\|\Sigma_{S}\right\|_{\infty, 1}$ depends on the forward DP, which itself is difficult to analyze. However, in Section 6, we offer empirical evidence that $\left\|\Sigma_{S}\right\|_{\infty, 1}$ scales with the conductance of $S$, a common measure of the clustering of $S$ in the underlying graph (see (22) for a formal definition). Furthermore, as will be discussed next, this quantity provably scales with clustering for the stochastic block model, a common model for networks with community structure. In short, when $S$ is clustered in the graph, $\left\|\Sigma_{S}\right\|_{\infty, 1}$ is typically small, and estimating PPR for many sources is easier.

We now turn to our result for the stochastic block model. We consider the special case for which $n$ is a perfect square and the graph is composed of $\sqrt{n}$ communities, each containing $\sqrt{n}$ nodes. (This allows us to compare the extremes of choosing $\sqrt{n}$ sources from the same community or from distinct communities; however, the analysis can be modified for other cases.) More specifically, we define $V_{n, i}=\{1+(i-1) \sqrt{n}, \ldots, i \sqrt{n}\}$ and set $V_{n}=\cup_{i=1}^{\sqrt{n}} V_{n, i}$; we will view each $V_{n, i}$ as a community. For $v \in V_{n}$, we denote by $i(v)$ the unique $i \in\{1, \ldots, \sqrt{n}\}$ satisfying $v \in V_{n, i}$ (i.e., $i(v)$ is the community that $v$ belongs to). We then construct a graph $G_{n}=\left(V_{n}, E_{n}\right)$ as follows: for any $u, v \in V_{n}$ subject to $u \neq v$, edge $u \rightarrow v$ is present with probability $p_{n}$ if $i(u)=i(v)$ (i.e., if $u, v$ are in the same community) and is present with probability $q_{n}$ if $i(u) \neq i(v)$ (i.e., if $u, v$ are in different communities), independent of other edges. We also define

$$
d_{\text {out }}(v)=\left|\left\{u \in V_{n}: v \rightarrow u \in E_{n}\right\}\right|, \quad d_{\text {out }}^{-}(v)=\left|\left\{u \in V_{n} \backslash V_{n, i(v)}: v \rightarrow u \in E_{n}\right\}\right| \quad \forall v \in V_{n} .
$$

In words, $d_{\text {out }}(v)$ is $v$ 's out-degree (as before, although here it is a random variable), and $d_{\text {out }}^{-}(v)$ is the number of edges pointing from $v$ to other communities.

Our analysis will assume $p_{n}=p$ is a constant and $q_{n}=o(1 / \sqrt{n})$. In this case, $\mathbb{E}\left[d_{\text {out }}(v)\right]=\Theta(\sqrt{n})$ (i.e., the graph is dense) and $\mathbb{E}\left[d_{\text {out }}^{-}(v)\right]=o(\sqrt{n})$ (i.e., nodes prefer to connect to their own community). In addition, we assume the forward DP is run for at most $o(\sqrt{n})$ iterations. Since all nodes have out-degree $\Theta(\sqrt{n})$ with high probability (see proof of Theorem 5.2), this means we dedicate at most $o(n)$ complexity to the forward DP. This is consistent with the fact that our algorithm has average-case complexity $O(\sqrt{m})$, since $\sqrt{m}=n^{3 / 4}$ when all out-degrees are $\Theta(\sqrt{n})$. Hence, this assumption on the number of iterations is minor. Under these assumptions, we can prove the following. 
TheOrem 5.2. Let $\left\{G_{n}=\left(V_{n}, E_{n}\right)\right\}_{n \in \mathbb{N}: \sqrt{n} \in \mathbb{N}}$ be the sequence of stochastic block models described earlier, with $p_{n}=p$ for some constant $p \in(0,1)$ and $q_{n}=o(1 / \sqrt{n})$. Assume we run the forward DP for at least one iteration but at most o $(\sqrt{n})$ iterations. Then the following hold:

- For each $n$, let $S_{n}=V_{n, i}$ for some $i \in\{1, \ldots, \sqrt{n}\}$ (i.e., all sources belong to the same community). If $q_{n}=\Omega(\log n / n)$ (i.e., cross-community connections are dense), then for some constant $C>0$,

$$
\lim _{n \rightarrow \infty} \mathbb{P}\left(\left\|\Sigma_{S_{n}}\right\|_{\infty, 1} \leq C q_{n} n\right)=1 \text {-that is, }
$$

$\left\|\Sigma_{S_{n}}\right\|_{\infty, 1}=O\left(q_{n} n\right)=o(\sqrt{n})$ with high probability. If instead $q_{n}=\Theta(1 / n)$ (i.e., crosscommunity connections are sparse), then for some constant $C>0$,

$$
\lim _{n \rightarrow \infty} \mathbb{P}\left(\left\|\Sigma_{S_{n}}\right\|_{\infty, 1} \leq C \log n / \log \log n\right)=1 \text {-that is, }
$$

$\left\|\Sigma_{S_{n}}\right\|_{\infty, 1}=O(\log n / \log \log n)$ with high probability.

- For each $n$, let $S_{n} \subset V_{n}$ with $\left|S_{n}\right|=\sqrt{n}$ and $i(s) \neq i\left(s^{\prime}\right) \forall s, s^{\prime} \in S_{n}$ subject to $s \neq s^{\prime}$ (i.e., each source belongs to a distinct community). Then for any constant $\delta \in(0,1)$,

$$
\lim _{n \rightarrow \infty} \mathbb{P}\left(\left\|\Sigma_{S_{n}}\right\|_{\infty, 1} \geq(1-\delta) \sqrt{n}\right)=1 \text {-that is, }
$$

$\left\|\Sigma_{S_{n}}\right\|_{\infty, 1} \in[(1-\delta) \sqrt{n}, \sqrt{n}]$ with high probability.

Proof. See Appendix H in Vial and Subramanian [29].

5.1.2 Target Stage Acceleration. Our next goal is to accelerate the target stage of the many pair estimation approach. For this, we propose a unified DP stage that avoids repeated computations that may occur when running backward DP for each target separately. We motivate our approach in the simple case $T=\left\{t_{1}, t_{2}\right\}$. Assume that $p^{t_{1}}, r^{t_{1}}$ have been computed by Algorithm 2 , and that $p^{t_{2}}, r^{t_{2}}$ are currently being computed. If $r^{t_{2}}\left(t_{1}\right)>r_{\max }^{t}$ at some iteration, we can use the alternate update rule given by (13) (rather than that given in Algorithm 2):

$$
p^{t_{2}} \leftarrow p^{t_{2}}+r^{t_{2}}\left(t_{1}\right) p^{t_{1}}, \quad r^{t_{2}} \leftarrow r^{t_{2}}+r^{t_{2}}\left(t_{1}\right)\left(r^{t_{1}}-e_{t_{1}}\right) .
$$

When $p^{t_{2}}, r^{t_{2}}$ are updated via (13), the invariant (6) is maintained. Indeed, for any $s \in V$,

$$
\begin{aligned}
& p^{t_{2}}(s)+r^{t_{2}}\left(t_{1}\right) p^{t_{1}}(s)+\sum_{u \in V} \pi_{s}(u)\left(r^{t_{2}}(u)+r^{t_{2}}\left(t_{1}\right)\left(r^{t_{1}}(u)-e_{t_{1}}(u)\right)\right) \\
& =p^{t_{2}}(s)+\sum_{u \in V} \pi_{s}(u) r^{t_{2}}(u)+r^{t_{2}}\left(t_{1}\right)\left(\left(p^{t_{1}}(s)+\sum_{u \in V} \pi_{s}(u) r^{t_{1}}(u)\right)-\pi_{s}\left(t_{1}\right)\right), \\
& =\pi_{s}\left(t_{2}\right)+r^{t_{2}}\left(t_{1}\right)\left(\pi_{s}\left(t_{1}\right)-\pi_{s}\left(t_{1}\right)\right)=\pi_{s}\left(t_{2}\right),
\end{aligned}
$$

where in (14) we simply rearranged terms and in (15) we assume $p^{t_{1}}, r^{t_{1}}$ and $p^{t_{2}}, r^{t_{2}}$ satisfy (6).

We can interpret the update rule (13) as follows. As discussed in Section 4, we view Algorithm 2 as a method of traversing paths to $t$ and computing the probability of these paths. For the update in Algorithm 2, specific paths are extended by a single step at each iteration; we call this update Extend. In contrast, the alternate update rule (13) extends paths by (potentially) many steps in an iteration, specifically by appending paths to $t_{1}$, with paths from $t_{1}$ to $t_{2}$, to obtain paths to $t_{2}$. We call this update Merge to highlight that longer paths are combined to obtain new paths.

The utility of Merge is that the probability of paths to $t_{2}$ through $t_{1}$ need not be recomputed one step at a time via Extend. This is depicted in Figure 3(b): red paths are computed via Extend during $t_{2}$ DP; blue paths, having already been computed via Extend during $t_{1} \mathrm{DP}$, are used to compute 
longer paths in a single iteration via Merge during $t_{2}$ DP. In contrast, blue paths would be recomputed one step at a time via Extend during $t_{2}$ DP, if separate DP was used. In short, Merge may allow Algorithm 2 to terminate in fewer iterations. This is made more specific in Proposition 5.3.

Proposition 5.3. Suppose $T=\left\{t_{1}, t_{2}\right\}$ and $\pi_{t_{1}}\left(t_{2}\right)>r_{\max }^{t}$. If we run Algorithm 2 for $t_{2}$ and use Merge at iterations for which $v^{*}=t_{1}$, the algorithm terminates in at most $\frac{n \pi\left(t_{2}\right)}{\alpha r_{\max }^{t}}-\frac{\left(\left\|p^{t_{1}}\right\|_{1}-\alpha\right)}{\alpha}$ iterations. If Merge is not used, the number of iterations for termination is at most $\frac{n \pi\left(t_{2}\right)}{\alpha r_{\max }^{t}}$.

Proof. See Appendix F.

From Algorithm 2, $\left\|p^{t_{1}}\right\|_{1} \geq \alpha$. Hence, Proposition 5.3 allows us to tighten the iteration bound by $\frac{\left(\left\|p^{t_{1}}\right\|_{1}-\alpha\right)}{\alpha} \geq 0$ (with equality if and only if the algorithm terminates in a single iteration for $t_{1}$ ). In the more general case, the iterations we save roughly scale with the quantity

$$
c_{T}=\sum_{i=1}^{|T|}\left|\left\{j \in\{1,2, \ldots, i-1\}: \pi_{t_{j}}\left(t_{i}\right)>r_{\max }^{t}\right\}\right|,
$$

assuming the nodes in $T$ are chosen in order $\left\{t_{1}, t_{2}, \ldots, t_{|T|}\right\}$. We note the choice of this order has a clear impact on performance, but optimizing it at runtime is difficult; we discuss this more in Appendix I in Vial and Subramanian [29]. See Algorithm 4 for our many target algorithm.

We next offer a clustering interpretation of the quantity $c_{T}$. For this, note $\pi_{t_{j}}\left(t_{i}\right)>r_{\max }^{t}$ is a notion of "closeness" between $t_{i}$ and $t_{j}$; hence, $c_{T}$ is a notion of clustering of the set $T$, and our analysis suggests estimating PPR for many targets is easier when the targets are clustered. Note that although the source clustering quantity $\|\Sigma\|_{\infty, 1}$ from Section 5.1.1 is smaller when clustering among sources is more significant, the target clustering quantity $c_{T}$ is larger when clustering among targets is more significant; in Section 6, we show $-c_{T}$ scales with the conductance of $T$ in practice.

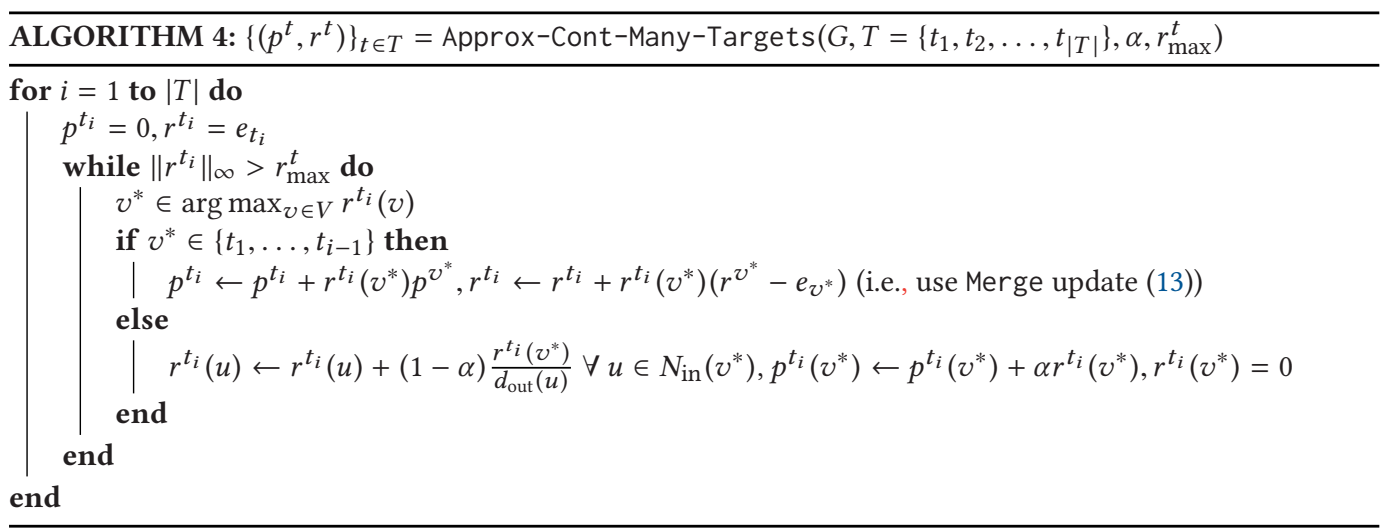

\subsection{Matrix Approximation Viewpoint}

For the second variant of the many pair estimation problem, we view $\left\{\pi_{S}(t)\right\}_{s \in S, t \in T}$ as a matrix that we aim to accurately approximate. For simplicity, we assume $|S|=|T|=l$, and we denote these sets $S=\left\{s_{i}\right\}_{i=1}^{l}, T=\left\{t_{i}\right\}_{i=1}^{l}$. We also assume $V=\{1,2, \ldots, n\}$, and we let $\Pi$ denote the matrix of dimension $n \times n$ whose $(i, j)$-th element is $\pi_{i}(j)$. In this notation, we seek an estimate $\hat{\Pi}(S, T)$ of $\Pi(S, T)$ that minimizes $\|\hat{\Pi}(S, T)-\Pi(S, T)\|_{2}$, where for a matrix $A, A(I, J)$ denotes the submatrix of $A$ containing rows $I$ and columns $J$, and where $\|A\|_{2}=\max _{\|x\|_{2}=1}\|A x\|_{2}$ is the operator norm. 
Before proceeding, we introduce additional notation used in this section. Similar to the $A(I, J)$ notation, $A(I,:)$ and $A(:, J)$ are the submatrices with rows $I$ and all columns, and all rows and columns $J$, respectively. For a vector $x, x(I)$ is the vector with elements $I$; when $x$ has nonzero entries, $\operatorname{diag}(x)$ and $\operatorname{diag}(1 / x)$ are the diagonal matrices whose $i$-th diagonal elements are $x(i)$ and $1 / x(i)$, respectively. Finally, we will encounter stable rank, which for a matrix $A$ is defined as $\operatorname{srank}(A)=\left(\|A\|_{F} /\|A\|_{2}\right)^{2}$, where $\|\cdot\|_{F}=\|\cdot\|_{2,2}$ is the Frobenius norm, with $\|\cdot\|_{2,2}$ defined as in (12). It is straightforward to verify $1 \leq \operatorname{srank}(A) \leq \operatorname{rank}(A)$ by writing $\|A\|_{F}^{2}$ and $\|A\|_{2}^{2}$ in terms the singular values of $A$ (e.g., see Section 2.1.15 of Tropp [28]).

With this notation in mind, we define the following matrices:

$$
\begin{aligned}
& P_{S} \in \mathbb{R}^{n \times l} \text { subject to } P_{S}(i, j)=p^{s_{j}}(i), \quad R_{S} \in \mathbb{R}^{n \times l} \text { subject to } R_{S}(i, j)=r^{s_{j}}(i), \\
& P_{T} \in \mathbb{R}^{n \times l} \text { subject to } P_{T}(i, j)=p^{t_{j}}(i), \quad R_{T} \in \mathbb{R}^{n \times l} \text { subject to } R_{T}(i, j)=r^{t_{j}}(i) .
\end{aligned}
$$

Here, $p^{s_{j}}, r^{s_{j}}$ and $p^{t_{j}}, r^{t_{j}}$ are assumed to have been computed via Algorithms 1 and 4 , respectively. We may then collect the invariant (7) for each $\left(s_{i}, t_{j}\right)$ pair in matrix form as

$$
\Pi(S, T)=P_{T}(S,:)+P_{S}^{\mathrm{T}} R_{T}+R_{S}^{\mathrm{T}} \Pi R_{T} .
$$

Observe only $R_{S}^{\top} \Pi R_{T}$ is unknown in (19). Hence, we consider estimation of this term. To this end, let $\sigma$ be any $n$-length vector satisfying $\sigma(i)>0 \forall i \in\{1,2, \ldots, n\}$ and $\sum_{i=1}^{n} \sigma(i)=1$; note we may view $\sigma$ as a distribution on $V$. We then rewrite the unknown term in (19) as

$$
R_{S}^{\mathrm{T}} \Pi R_{T}=R_{S}^{\mathrm{T}} \operatorname{diag}(1 / \sigma) \operatorname{diag}(\sigma) \Pi R_{T} .
$$

Using (20), we can obtain unbiased estimates of $R_{S}^{\mathrm{T}} \Pi R_{T}$ as follows. Let $\left\{\mu_{i}\right\}_{i=1}^{w}$ be independent and identically distributed samples from $\sigma$. For $i \in\{1,2, \ldots, w\}$, let $v_{i} \sim \pi_{\mu_{i}}$ independently (where we sample from $\pi_{\mu_{i}}$ using a random walk, as given by (3)), and let $X_{i}=R_{S}^{\top} \operatorname{diag}(1 / \sigma) e_{\mu_{i}} e_{v_{i}}^{\top} R_{T}$. It is straightforward to see $\mathbb{E}\left[e_{\mu_{i}} e_{v_{i}}^{\top}\right]=\operatorname{diag}(\sigma) \Pi$; hence, $\mathbb{E}\left[X_{i}\right]=R_{S}^{\mathrm{T}} \Pi R_{T}$. We may then estimate $\Pi(S, T)$ as $\hat{\Pi}(S, T)=P_{T}(S,:)+P_{S}^{\top} R_{T}+\frac{1}{w} \sum_{i=1}^{w} X_{i}$.

We will consider two forms of $\sigma$ for this approach. Specifically, let us define

$$
\sigma_{\mathrm{avg}}(i)=\frac{1}{l} \sum_{s \in S} \sigma_{s}(i), \quad \sigma_{\max }(i)=\frac{1}{\left\|\sum\right\|_{\infty, 1}} \max _{s \in S} \sigma_{s}(i),
$$

where $\sigma_{s}=r^{s} /\left\|r^{s}\right\|_{1}$ as before. Observe that when $\sigma \in\left\{\sigma_{\mathrm{avg}}, \sigma_{\max }\right\}$, the assumption $\sum_{i=1}^{n} \sigma(i)=1$ is satisfied. Furthermore, we argue that the assumption $\sigma(i)>0$ is without loss of generality in these cases. Indeed, suppose $\sigma(j)=0$ for some $j$ and $\sigma(i)>0$ for $i \neq j$. Then $\mathbb{P}\left[\mu_{i}=j\right]=0$ by definition, and by (21), $r^{s}(j)=0 \forall s \in S$. It is then readily verified that $R_{S}(V \backslash\{j\},:)^{\top} \operatorname{diag}(1 / \sigma(V \backslash$ $\{j\})) e_{\mu_{i}} e_{v_{i}}^{\mathrm{T}} R_{T}$ is an unbiased estimate of $R_{S}^{\mathrm{T}} \Pi R_{T}$. Given this simple fix, we assume $\sigma(i)>0$ moving forward.

To summarize, we have proposed the matrix approximation scheme formally defined in Algorithm 5. Theorem 5.4 provides a guarantee for the accuracy of this scheme.

Theorem 5.4. Fix $\epsilon>0$. If $\sigma=\sigma_{\text {avg }}$ in Algorithm 5, assume the number of walks $w$ satisfies

$$
w \geq l^{2} \sqrt{\operatorname{srank}(\Pi(S, T))} \log \left(2 l / p_{\text {fail }}\right) r_{\max }^{s} r_{\max }^{t}(6+4 \epsilon) /\left(3 \epsilon^{2}\right) .
$$

If instead $\sigma=\sigma_{\max }$ in Algorithm 5, assume w satisfies

$$
w \geq l^{3 / 2}\|\Sigma\|_{\infty, 1} \log \left(2 l / p_{\text {fail }}\right) r_{\max }^{s} r_{\max }^{t}(6+4 \epsilon) /\left(3 \epsilon^{2}\right) .
$$

Then for both choices of $\sigma$, and with probability at least $1-p_{\text {fail }}$, Algorithm 5 returns an estimate $\hat{\Pi}(S, T)$ satisfying $\|\Pi(S, T)-\hat{\Pi}(S, T)\|_{2} \leq \epsilon \max \left\{\|\Pi(S, T)\|_{2}, 1\right\}$. 


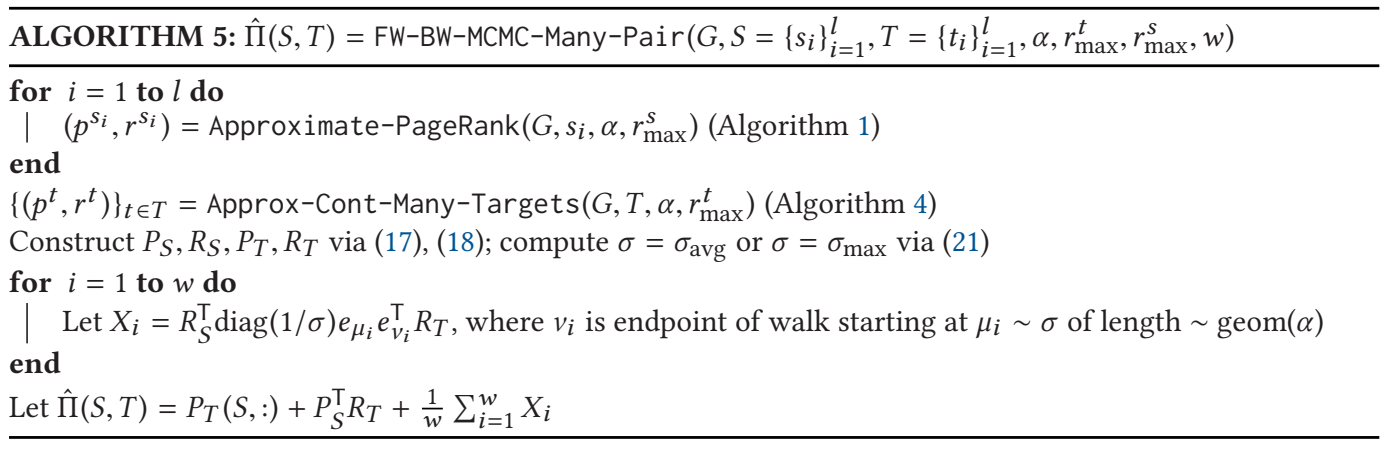

Proof. See Appendix G.

We note that, neglecting common factors, Theorem 5.4 states $w$ scales with $l^{2}$ and $l^{3 / 2}$ in the best case for $\sigma_{\text {avg }}$ and $\sigma_{\max }$, respectively; in the worst case, $w$ scales with $l^{5 / 2}$ for both approaches. In the next section, we compare $\sqrt{l \operatorname{srank}(\Pi(S, T))}$ with $\|\Sigma\|_{\infty, 1}$ empirically to compare the "typical" case.

Next, we observe Theorem 5.4 shows that, as in the scalar estimation viewpoint of Section 5.1, PPR matrix approximation is easier when clustering occurs. This is because when $\sigma=\sigma_{\max }$, complexity scales with $\|\Sigma\|_{\infty, 1}$ (which we have argued is measure of clustering of $S$ ); when $\sigma=\sigma_{\mathrm{avg}}$, complexity scales with $\operatorname{srank}(\Pi(S, T))$, a measure of matrix dimensionality. Additionally, stable rank is unique from the clustering quantities introduced thus far in that it takes into account both $S$ and $T$ (unlike $\|\Sigma\|_{\infty, 1}$, which only accounts for $S$, or $c_{T}$, which only accounts for $T$ ).

Finally, we comment on a difference for the choices of $\sigma$. In particular, when $\sigma=\sigma_{\max }$, one can set $w$ proportional to $\|\Sigma\|_{\infty, 1}$ before sampling random walks, leveraging clustering at runtime to increase efficiency. In contrast, when $\sigma=\sigma_{\text {avg }}$, the scaling factor in the $w$ lower bound is the unknown quantity $\operatorname{srank}(\Pi(S, T))$. However, we propose using $\operatorname{srank}\left(P_{T}(S,:)+P_{S}^{\mathrm{T}} R_{T}\right)($ known at runtime) as a surrogate for $\operatorname{srank}(\Pi(S, T))$. In Section 6, we show empirically that using this surrogate yields performance similar to using $\operatorname{srank}(\Pi(S, T))$.

\subsection{Precomputation Variants}

Although we have thus far assumed all computations are done online, one can also consider variants for which some computations are done offline, with the results stored for later use. In fact, in Section 4 of Lofgren et al. [20], the authors propose several such algorithms for the case of one source $s \in V$ and many targets $T \subset V$, using Bidirectional-PPR as a primitive. Each of these variants proceeds as follows. For the offline stage, Approx-Contributions is run for every $t \in V$, and the vectors $\left\{p^{t}, r^{t}\right\}_{t \in V}$ are stored. For the online stage, random walks are sampled from $s$, and $\left\{\pi_{s}(t)\right\}_{t \in T}$ are estimated using the endpoints of these walks and $\left\{p^{t}, r^{t}\right\}_{t \in T}$. As mentioned, several such algorithms are proposed; these only differ in how the vectors are stored and how the walks and vectors are combined to generate estimates. In particular, the basic framework of running Approx-Contributions offline and sampling walks from $s$ online is used in all of the precomputation algorithms from Lofgren et al. [20].

Analogous to our extension of Bidirectional-PPR from the single pair case to the many pairs case, we can extend these precomputation algorithms from the single source case to the many sources case. Specifically, we can modify each of these algorithms in two ways (but otherwise leave them unchanged). First, we can modify the offline stage by also precomputing and storing $\left\{p^{s}, r^{s}\right\}_{s \in V}$ via Approx-PageRank. Second, we can modify the online stage by sampling walks using the precomputed vectors $\left\{r^{s}\right\}_{s \in S}$ and the walk sharing scheme from Section 5.1.1. 

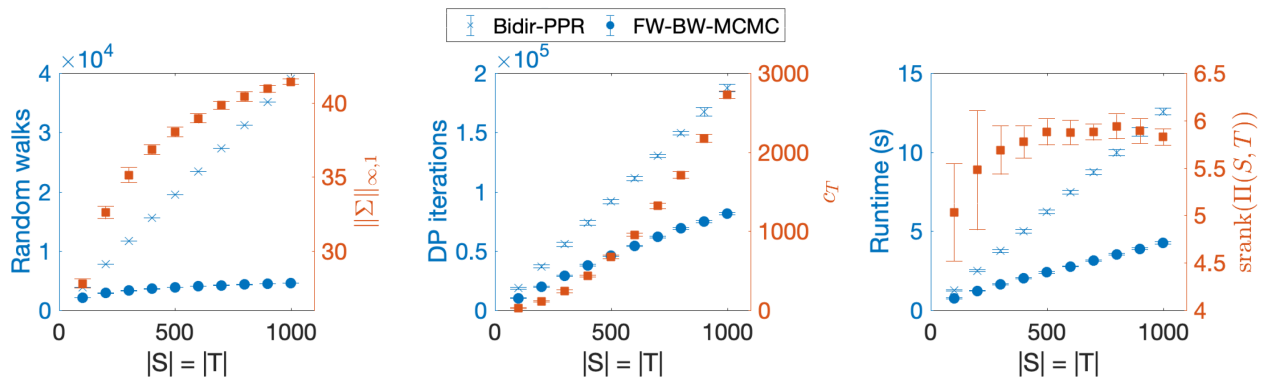

Fig. 4. On Direct-ER, random walks, backward DP iterations, and runtime scale more slowly in $|S|,|T|$ for our method FW-BW-MCMC when compared to the existing method Bidir-PPR.

To assess the performance of this approach, we compare against the naive extension of Lofgren et al.'s [20] precomputation algorithms to the case $|S|>1$, namely leaving the offline stage unchanged and sampling walks separately from each $s \in S$ online. Clearly, our approach requires more storage (due to running Approx-PageRank offline); however, this storage will be roughly double that of the naive extension and thus will not increase the order of the space complexity. However, our approach will accelerate the online stage of this naive extension, since fewer random walks will typically be sampled. Specifically, per Section 5.1.1, we expect to sample $w\|\Sigma\|_{\infty, 1}$ walks instead of $w|S|$ walks; as discussed previously, the former quantity can be much smaller if $S$ is clustered.

We also note that Algorithm 4 can be used to compute $\left\{p^{t}, r^{t}\right\}_{t \in V}$ offline, although this is a minor point, since offline computational complexity is generally not a concern. However, this raises another point. When precomputation is not allowed, our source and target accelerations are both used at runtime; when precomputation is allowed, only our source acceleration is used at runtime. Hence, the runtime savings of our schemes may be less significant in the precomputation setting. Despite this, we believe the savings will still be considerable in general. This belief follows from the fact that, in our experiments, the source acceleration is generally at least as significant as the target acceleration. For example, Figure 4 shows that the number of random walks sampled grows more slowly in $|S|$ than the number of DP iterations grows in $|T|$. Additionally, later, Figure 7 shows that for fixed $|S|,|T|$, walk savings and DP iteration savings are comparable across a wide range of graphs.

\section{EXPERIMENTS}

In this section, we demonstrate the empirical performance of our algorithms and the role of clustering in their performance. We conduct experiments using both synthetic and real graphs. On the synthetic side, we use a directed Erdős-Rényi graph and directed stochastic block model (referred to hereafter as Direct-ER and Direct-SBM, respectively), each with $n=2 \times 10^{3}$ and $\mathbb{E}[\mathrm{m}]=$ $2 \times 10^{4}$. For the real datasets, we use a set of graphs from the Stanford Network Analysis Platform [19] including social networks (Slashdot, Wiki-Talk), partial Web crawls (web-BerkStan, web-Google), co-purchasing and co-authoring graphs (com-amazon, com-dblp), and a road network (roadNet-PA). In addition to the diverse domains of these datasets, they differ in terms of sparsity (in order of magnitude, each has $10^{6}$ edges, but the number of nodes ranges from $10^{4}$ to $10^{6}$ ), so we believe our empirical results are robust across different graph structures. We also note the error bars depict standard deviation across experimental trials, whereas for scatter plots without error bars, each dot represents a single trial. For further experimental documentation, we point the reader to Appendix J in Vial and Subramanian [29]. In particular, Table 3 in Vial 
and Subramanian [29] documents algorithmic parameters used. We chose these parameters so the primitive algorithms FW-BW-MCMC and Bidirectional-PPR yield similar accuracy $(\approx 10 \%$ relative error) while balancing runtime between the DP and MCMC stages of the algorithm in the single pair case. Note the analysis in Appendix A shows that balancing runtime in this manner minimizes overall complexity; hence, for both algorithms, our chosen parameters optimize runtime subject to an accuracy constraint, providing a fair comparison. Finally, the implementation of our algorithms is available at https://github.com/danielvial/clusteringPpr.

\subsection{Synthetic Data}

6.1.1 Scalar Estimation. We first compare FW-BW-MCMC with Bidirectional-PPR when computing $\pi_{s}(t) \forall(s, t) \in S \times T$ as $|S|$ and $|T|$ grow on Direct-ER. More specifically, for FW-BW-MCMC, we use the $\left\|D^{-1} r^{s}\right\|_{\infty} \leq r_{\max }^{s}$ forward DP scheme as in FW-BW-MCMC-Practical, sample walks using the scheme from Section 5.1.1, and Algorithm 4 for backward DP; for Bidirectional-PPR, we sample walks separately from each $s \in S$ and run backward DP separately for each $t \in T$. Results are shown in Figure 4. Note the number of random walks sampled and number of backward DP iterations grow more slowly with $|S|=|T|$ using FW-BW-MCMC, due to the accelerations proposed in Sections 5.1.1 and 5.1.2, respectively. As a result, runtime grows more slowly using FW-BW-MCMC. In Figure 4, we also show the clustering quantities (11) and (16). We observe the source clustering quantity $\|\Sigma\|_{\infty, 1}$ has a concave shape, which corresponds to the apparent sublinear growth of random walks as $|S|$ grows. Additionally, the target clustering quantity $c_{T}$ has a convex shape; since backward DP iteration savings scale with $c_{T}$, we expect DP iterations to correspondingly "flatten," which indeed occurs. These observations empirically validate the key insights of Section 5.1, namely that the estimation schemes proposed have complexities that scale with the identified clustering quantities $\|\Sigma\|_{\infty, 1}$ and $c_{T}$. We also plot $\operatorname{srank}(\Pi(S, T))$ on the runtime plot; note it appears to flatten along with runtime as $|S|,|T|$ grow. Finally, these plots remain qualitatively similar as $n$ grows, whereas the improvement of our scheme over the existing one increases; see Appendix J in Vial and Subramanian [29].

Next, to further examine the effect of clustering, we use Direct-SBM. We fix $|S|=|T|=100$ and sample $S$ and $T$ from decreasingly clustered sets via the following scheme: we first sample $S, T$ from a single community, we then sample $S, T$ from two communities, and so forth, until we sample $S, T$ from the entire graph, allowing us to observe a wide range of clustering. As in the previous experiment, we are interested in how algorithmic performance relates to $\|\Sigma\|_{\infty, 1}$ and $c_{T}$. Here, we also compare these quantities to a clustering measure commonly used in the graph theory literature (e.g., see the aforementioned [2]), conductance, defined for $U \subset V$ as

$$
\Phi(U)=\frac{\sum_{i \in U, j \notin U} A_{i j}}{\min \left\{\sum_{u \in U} d_{\text {out }}(u), \sum_{u \notin U} d_{\text {out }}(u)\right\}} .
$$

In Figure 5, we observe fewer random walks are sampled when $\Phi(S)$ is small (when $S$ is significantly clustered); similarly, the backward DP converges in fewer iterations when $\Phi(T)$ is small (when $T$ is significantly clustered). Furthermore, Figure 5 shows that $\|\Sigma\|_{\infty, 1}$ grows with $\Phi(S)$ and $-c_{T}$ grows with $\Phi(T)$. In short, our identified clustering quantities behave similar to conductance. In the runtime plot, we again show $\operatorname{srank}(\Pi(S, T))$ as a measure of overall complexity; this quantity (roughly) grows with the average conductance $\frac{1}{2}(\Phi(S)+\Phi(T)$ ), as does runtime.

6.1.2 Matrix Approximation. We now document performance of our matrix approximation scheme (Algorithm 5) using Direct-SBM and the $S, T$ sampling strategy from the previous experiment. We compare three cases: $\sigma=\sigma_{\max }$ with $w \propto\|\Sigma\|_{\infty, 1}, \sigma=\sigma_{\text {avg }}$ with $w \propto$ $\sqrt{l \operatorname{srank}(\Pi(S, T))}$, and $\sigma=\sigma_{\text {avg }}$ with $w \propto \sqrt{l \operatorname{srank}\left(P_{T}(S,:)+P_{S}^{\mathrm{T}} R_{T}\right)}$. These cases are motivated by 

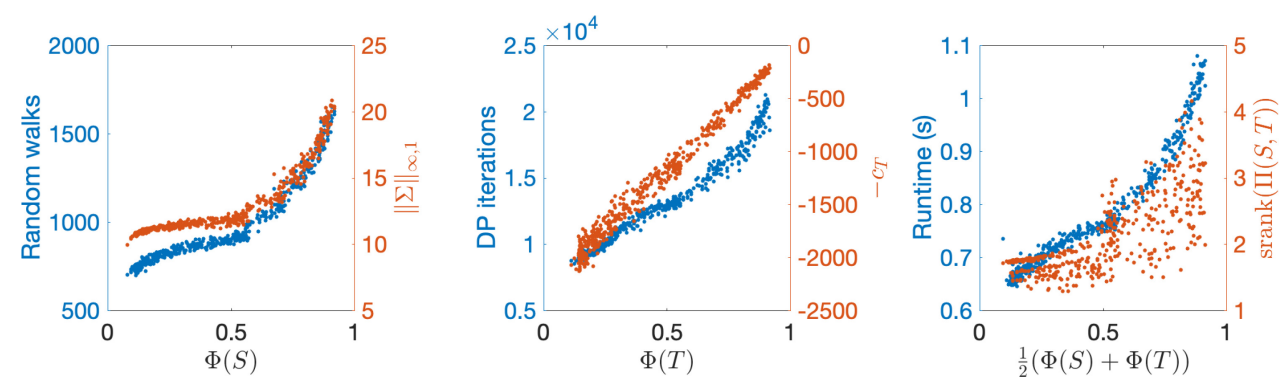

Fig. 5. When clustering is significant, fewer random walks and backward DP iterations yield faster runtime for our method on Direct-SBM; additionally, our clustering measures roughly scale with conductance.
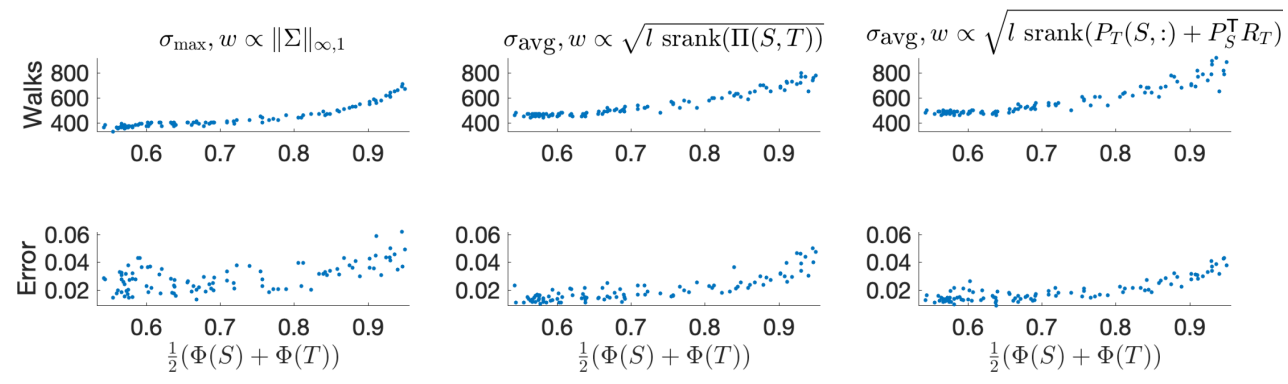

Fig. 6. On Direct-SBM, our matrix approximation schemes are most efficient when clustering is significant; additionally, the surrogate $\left.\operatorname{srank}\left(P_{T}(S,:)+P_{S}^{\mathrm{T}} R_{T}\right)\right)$ performs similar to $\operatorname{srank}(\Pi(S, T))$.

Theorem 5.4, which states that the sample requirements for $\sigma=\sigma_{\max }$ and $\sigma=\sigma_{\text {avg }}$ are $\|\Sigma\|_{\infty, 1}$ and $\sqrt{l \operatorname{srank}(\Pi(S, T))}$, respectively (neglecting common factors); additionally, since $\operatorname{srank}(\Pi(S, T)$ ) is unknown in practice, we proposed using $\operatorname{srank}\left(P_{T}(S,:)+P_{S}^{\top} R_{T}\right)$ as a surrogate in the discussion following the theorem. Results are shown in Figure 6. Observe that for all three cases, fewer walks are sampled when $S$ and $T$ are clustered (i.e., when $\frac{1}{2}(\Phi(S)+\Phi(T)$ ) is small; nevertheless, error remains roughly constant (in fact, when clustering is present, error is somewhat lower despite fewer walks being sampled). Further, we observe $\sigma_{\max }$ and $\sigma_{\text {avg }}$ have similar performance, in terms of complexity and accuracy. Finally, we note the results for the $\operatorname{srank}(\Pi(S, T))$ and $\operatorname{srank}\left(P_{T}(S,:)+P_{S}^{\top} R_{T}\right)$ cases are quite similar, suggesting that $\operatorname{srank}\left(P_{T}(S,:)+P_{S}^{\top} R_{T}\right)$ is an appropriate surrogate for $\operatorname{srank}(\Pi(S, T))$.

\subsection{Real Data}

6.2.1 Scalar Estimation. We next compare FW-BW-MCMC with Bidirectional-PPR as in Section 6.1.1, but here we use real datasets. We fix $|S|=|T|=1,000$ and randomly sample $S, T$ using two different schemes: sampling uniformly among all nodes and using an algorithm described in Appendix J of Vial and Subramanian [29] to build clustered subsets of nodes; we find these schemes typically give conductance values $\approx 0.99$ and $\approx 0.5$, respectively, allowing us to observe two degrees of clustering. In Figure 7, we show random walk count, DP iteration count, and runtime for our method relative to the corresponding values using Bidirectional-PPR. Averaging across the diverse set of graphs considered, our method is approximately 1.4 times faster in the uniform case and 2.9 times faster in the clustered case, highlighting the efficiency of our algorithms and the impact of clustering on their performance. Additionally, we note our method is at least twice as fast for all datasets in the clustered case. For the same experiment, we also show random walk 

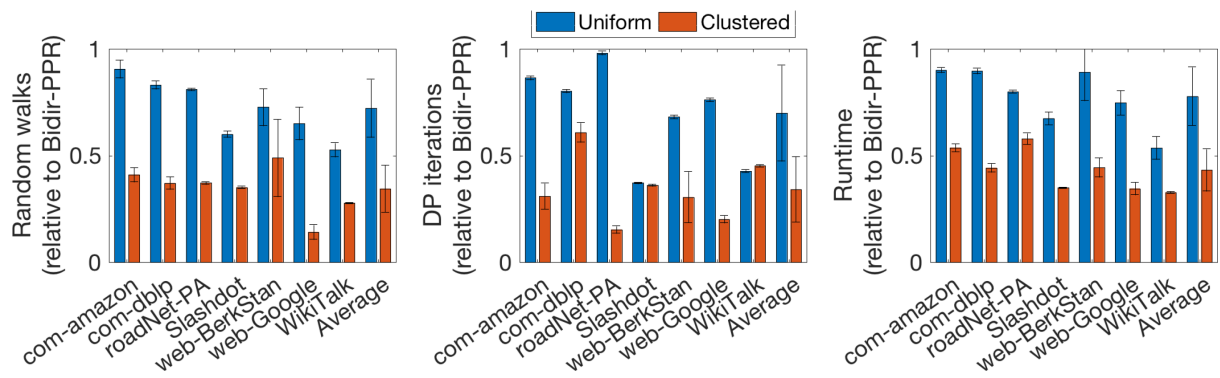

Fig. 7. On real graphs, our scalar methods are typically 1.4 and 2.9 times faster than existing methods when $S, T$ are chosen uniformly and clustered, respectively, due to fewer random walks and DP iterations.
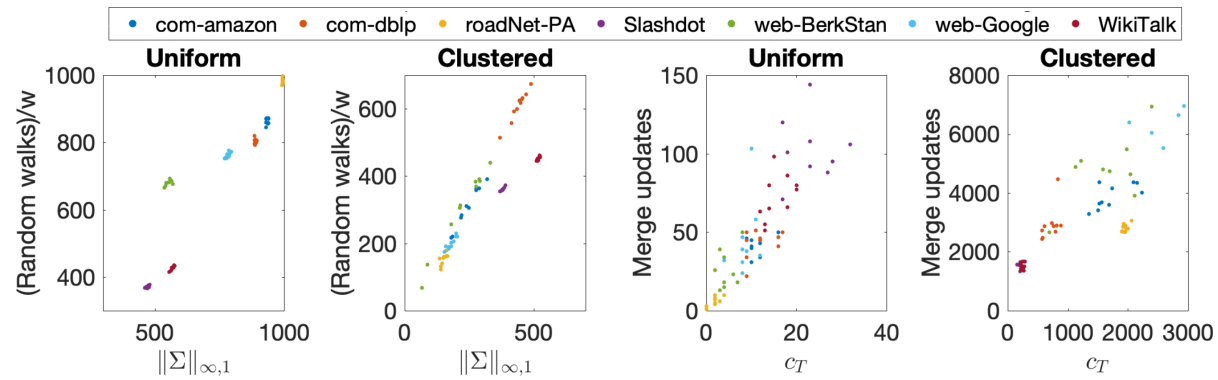

Fig. 8. On real graphs, random walks and Merge updates scale with clustering quantities $\|\Sigma\|_{\infty, 1}$ and $c_{T}$, empirically validating the analysis of Section 5.1 across diverse datasets.

count (normalized to $w$ ) and the number of Merge updates (i.e., the number of DP iterations saved when compared to existing methods) in Figure 8. From Theorem 5.1 and Proposition 5.3, we expect these quantities to scale linearly with the identified clustering quantities $\|\Sigma\|_{\infty, 1}$ and $c_{T}$, respectively; from Figure 8, we observe this scaling roughly occurs. This verifies our analysis empirically on real datasets.

6.2.2 Matrix Approximation. Finally, we test our matrix approximation scheme (Algorithm 5) on real graphs. Here, we also compare to a baseline method that does not leverage clustering among targets and sources. In particular, we run backward DP separately for each target rather than using the accelerated scheme as in Algorithm 5. Additionally, the baseline method uses no forward DP (i.e., we set $r_{\max }^{s}=1$ in Algorithm 5 so that $p^{s}=0, r^{s}=e_{s} \forall s \in S$ ). Note, in this case, both the $\sigma_{\max }$ and $\sigma_{\text {avg }}$ schemes reduce to sampling $\mu_{i} \sim S$ uniformly, sampling $v_{i} \sim \pi_{v_{i}}$ using a random walk, and estimating $\Pi(S, T)$ as $\hat{\Pi}(S, T)=P_{T}(S,:)+\frac{1}{w} \sum_{i=1}^{w} X_{i}$, where $X_{i}=\left[\begin{array}{llll}e_{s_{1}} & e_{s_{2}} & \cdots & e_{s_{l}}\end{array}\right]^{\top} e_{\mu_{i}} e_{v_{i}}^{\top} R_{T}$ is an unbiased estimate of $\Pi(S,:) R_{T}$. We reemphasize that walks are not shared among sources for this baseline scheme-that is, clustering among sources is not leveraged to improve performance. For the baseline scheme, we set $w \propto l$, and we compare performance to the $\sigma_{\max }$ scheme with $w \propto$ $\|\Sigma\|_{\infty, 1}$ and the $\sigma_{\text {avg }}$ scheme with $w \propto \sqrt{l \operatorname{srank}\left(P_{T}(S,:)+P_{S}^{\mathrm{T}} R_{T}\right)}$. Results are shown in Figure 9, with quantities shown for the $\sigma_{\max }$ and $\sigma_{\text {avg }}$ schemes relative to the baseline scheme. Averaging across datasets, the $\sigma_{\max }$ and $\sigma_{\text {avg }}$ schemes are over twice as fast as the baseline scheme when $S, T$ are chosen uniformly and 3.4 times faster when $S, T$ are clustered; additionally, the accuracy of both schemes is comparable to the baseline across datasets (and slightly better on average). We also note both our schemes are at least twice as fast as the baseline for all graphs in the clustered case. 

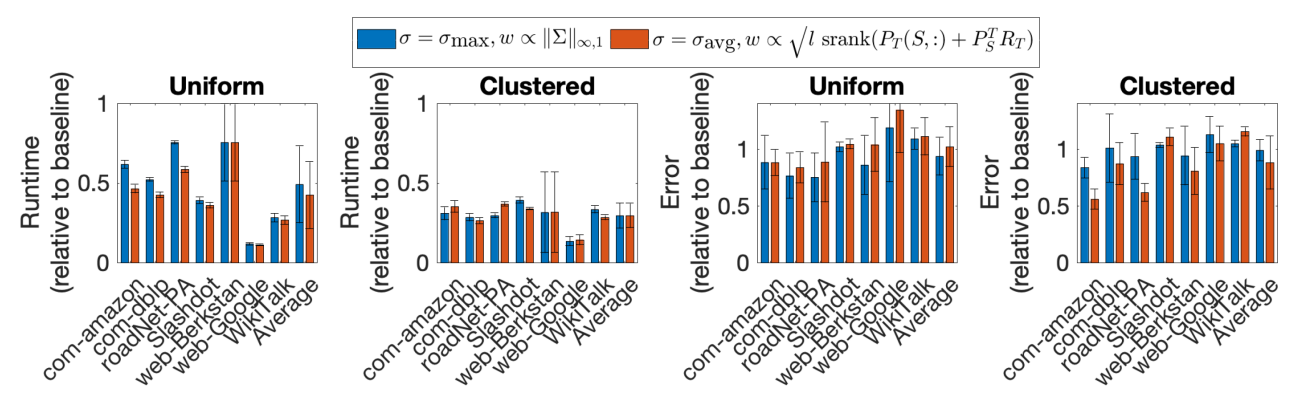

Fig. 9. On real graphs, our matrix approximation schemes are significantly faster than the baseline method (which uses no forward DP) with comparable accuracy; this is most notable when $S, T$ are clustered.

\section{APPLICATION: DISTRIBUTED RANDOM WALK SAMPLING}

Thus far, our key finding has been that PPR estimation complexity scales with quantities that describe clustering among sources and/or targets. In this section, we demonstrate one application of these findings, namely that these findings can be used to efficiently estimate $\left\{\pi_{s}\right\}_{s \in S}$ online when several machines are available and when offline storage is permitted. More specifically, we consider a natural distributed computational setting with the following features:

- $k$ machines are available for parallel computation and a central machine is available to facilitate the parallel computation (for simplicity, we assume $k \in\{|S|,|S| / 2,|S| / 3, \ldots\}$ ), and

- $\left\{p^{t}, r^{t}\right\}_{t \in V}$ have been precomputed via Algorithm 2 and are stored offline.

Using the existing method Bidirectional-PPR as a primitive, a baseline strategy for this estimation task is as follows: arbitrarily partition $S$ into $k$ subsets of size $|S| / k$, use the $i$-th machine to sample random walks from each source $s$ belonging to the $i$-th subset, and estimate $\pi_{s}$ using the endpoints of walks from $s$ and $\left\{p^{t}, r^{t}\right\}_{t \in V}$ (as in the primitive method Bidirectional-PPR).

Our goal is to devise a strategy more efficient than this baseline. In particular, we propose the following approach. First, we arbitrarily partition $S$ into $k$ subsets of size $|S| / k$, and we use the $i$-th machine to run forward DP (Algorithm 1) for each source $s$ belonging to the $i$-th subset. Second, we use the central machine to construct another partition $\left\{S_{i}\right\}_{i=1}^{k}$ of $S$, in a manner we discuss shortly. Third, we use the $i$-th machine to run the accelerated source stage from Section 5.1.1 for the subset of sources $S_{i}$. Finally, we estimate $\pi_{s}$ as in the primitive method FW-BW-MCMC.

It remains to specify how to construct the partition $\left\{S_{i}\right\}_{i=1}^{k}$. For this, we turn to Theorem 5.1 and the results of Section 6, which indicate the number of random walks sampled on the $i$-th machine scales with $\left\|\Sigma_{S_{i}}\right\|_{\infty, 1}$, where $\Sigma_{S_{i}}$ is the matrix with rows $\left\{\sigma_{s}\right\}_{s \in S_{i}}$. Hence, as the random walk stage in our approach runs in parallel across $i$, the runtime of the this stage scales with

$$
\max _{i \in\{1, \ldots, k\}}\left\|\Sigma_{S_{i}}\right\|_{\infty, 1} \text {. }
$$

Our goal is thus to construct the partition $\left\{S_{i}\right\}_{i=1}^{k}$ so as to minimize (23). However, as this is a combinatorial optimization problem, we devise a heuristic method to approximate the solution. To simplify the discussion of this method, we introduce some notation. For $S^{\prime} \subset S$, let $\sigma_{S^{\prime}}$ be subject to $\sigma_{S^{\prime}}(v)=\max _{s^{\prime} \in S^{\prime}} \sigma_{S^{\prime}}(v) \forall v \in V$; note $\left\|\Sigma_{S^{\prime}}\right\|_{\infty, 1}=\left\|\sigma_{S^{\prime}}\right\|_{1}$. For $S^{\prime} \subset S$ and $s \in S \backslash S^{\prime}$, let

$$
d\left(s, S^{\prime}\right)=\sum_{v \in V} \max \left\{\sigma_{s}(v)-\sigma_{S^{\prime}}(v), 0\right\}
$$

It is straightforward to show (25) (i.e., (24) gives the increase in $\left\|\sigma_{S^{\prime}}\right\|_{1}$ if $s$ is added to $S^{\prime}$ ):

$$
d\left(s, S^{\prime}\right)=\left\|\sigma_{S^{\prime} \cup\{s\}}\right\|_{1}-\left\|\sigma_{S^{\prime}}\right\|_{1} .
$$



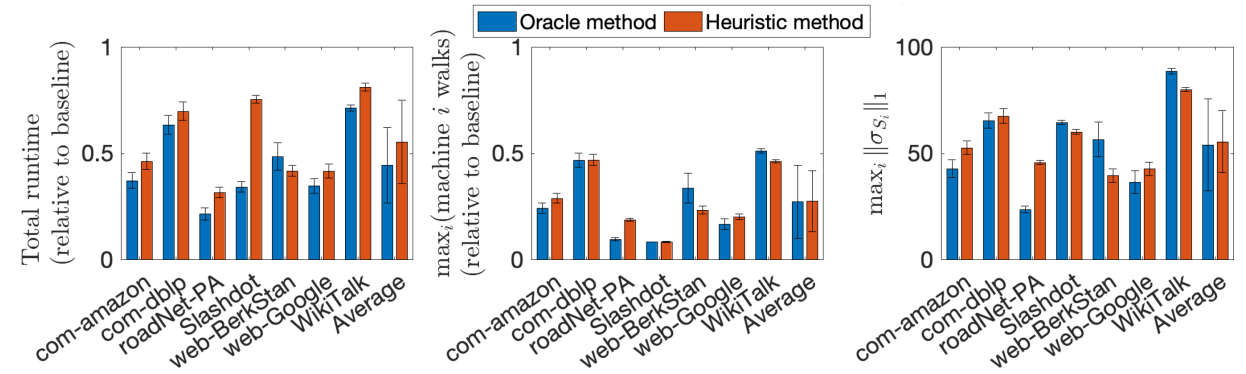

Fig. 10. In the distributed setting, our heuristic method is typically 1.8 times faster than the baseline, samples $\frac{1}{4}$ of the walks, and produces a low objective function value, with performance similar to an oracle method.

With this notation in place, we may restate the objective function (23) as

$$
\max _{i \in\{1, \ldots, k\}}\left\|\sigma_{S_{i}}\right\|_{1} .
$$

Our heuristic method to approximate the minimizer of (26) proceeds as follows. First, we assign one node to each $S_{i}, i \in\{1, \ldots, k\}$, using an initialization method similar to $k$-means++ [3]: we choose the $i$-th of these nodes with probability proportional to its distance from the first $(i-1)$ of them, in hopes of choosing initial nodes with $\sigma_{s}$ vectors far apart. Next, we iteratively assign the remaining $|S|-k$ nodes to some $S_{j}$. In particular, we assign $s$ to $S_{j}$ such that $d\left(s, S_{j}\right)+\left\|\sigma_{S_{j}}\right\|_{1}$ is minimal; from (25), this can be viewed as minimizing the increase in the objective function (26) incurred by assigning $s$ to some $S_{j}$. This heuristic method is formally defined in Algorithm 6 .

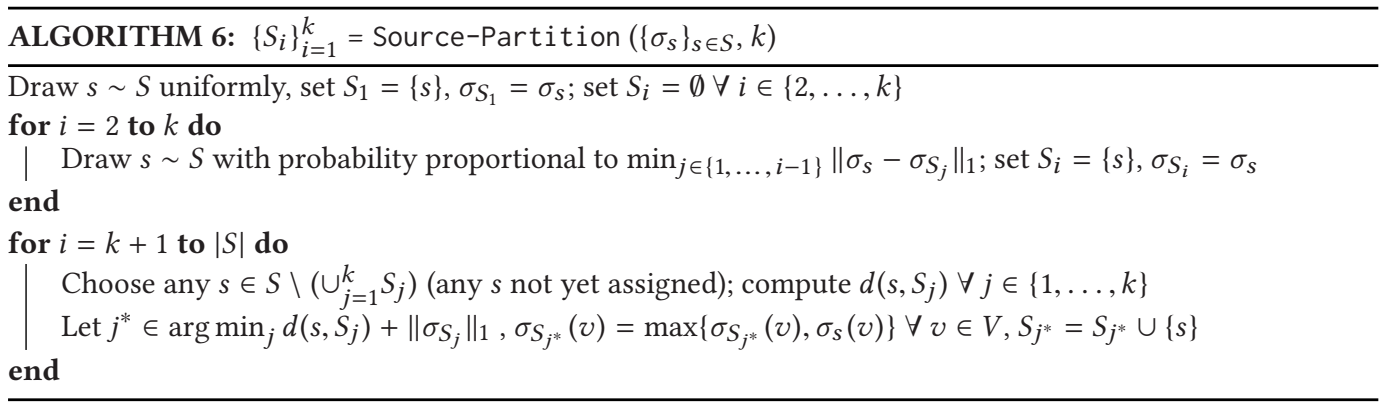

We now empirically compare our approach with the baseline scheme. For this experiment, we set $S=\left\{\tilde{S}_{i}\right\}_{i=1}^{k}$, where each $\tilde{S}_{i}$ is a clustered subset of nodes constructed as in Section 6 (with $k=10$ and $\left|\tilde{S}_{i}\right|=100 \forall i$ ). This yields a set of sources $S$ that is not highly clustered itself, but that contains $k$ subsets that are densely connected internally and sparsely connected to other subsets. In addition to comparing to the baseline, we also test the performance of an "oracle" scheme, which knows the clustering information of the input set $S$. More specifically, the oracle scheme proceeds in the same manner as our scheme, except instead of using Algorithm 6 to construct the partition $\left\{S_{i}\right\}_{i=1}^{k}$, it simply sets $S_{i}=\tilde{S}_{i} \forall i \in\{1,2, \ldots, k\}$. Put differently, whereas the heuristic scheme attempts to learn an assignment of sources to machines for which each machine is assigned a clustered set of sources (in the sense that (26) is minimal), the oracle scheme knows such an assignment a priori.

Results for this experiment are shown in Figure 10, using the set of real graphs from Section 6. Averaging across graphs, the oracle and heuristic methods are roughly 1.8 and 2.2 faster than the baseline scheme, respectively (left). (Here, total runtime is computed as maximum walk sampling 
time across machines for the baseline; sum of maximum forward DP time and maximum walk time for the oracle; and sum of maximum forward DP time, maximum walk time, and time to run Algorithm 6 for the heuristic.) Additionally, both methods sample approximately $\frac{1}{4}$ of the random walks sampled by the baseline scheme, across graphs (middle). Finally, the heuristic method typically produces a partition $\left\{S_{i}\right\}_{i=1}^{k}$ of $S$ with objective function value (26) similar to that produced by the oracle method (right). Interestingly, the heuristic outperforms the oracle for several datasets. This suggests the cluster information known by the oracle does not necessarily produce an optimal assignment of sources to machines; rather, the source clustering quantity $\left\|\sigma_{S_{i}}\right\|_{1}$ identified in Section 5.1.1 may be what truly dictates performance.

Before closing, we offer several remarks on this distributed setting. First, although we focused on the scalar estimation scheme from Section 5.1.1, the framework extends to the $\sigma_{\max }$ matrix approximation scheme from Section 5.2. In particular, using the latter scheme in this setting would also involve construction of a partition so as to minimize (26), per Theorem 5.4. For this reason, we expect the performance of this scheme to be similar to Figure 10. Second, we note using the $\sigma_{\text {avg }}$ matrix approximation scheme in this setting requires a partition that minimizes a different objective function. In Appendix K of Vial and Subramanian [29], we present an algorithm to construct such a partition, as well as empirical results describing performance (in short, our scheme performs similarly to the oracle and noticeably outperforms the baseline, as in Figure 10). Third, we find in practice that our heuristic partitioning schemes naturally balance the number of sources assigned to each machine (see Appendix K of Vial and Subramanian [29]). Such balance is crucial in the performance of our scheme. This is because we require $\left\|\sigma_{S_{i}}\right\|_{1}=O(|S| / k) \forall i$ to perform as well as the baseline, which may in turn require an extreme degree of clustering if the partition is unbalanced (e.g., if $\left|S_{i}\right|=O(|S|)$ for some $i$ ). It is worth noting that we also tried to partition $\left\{\sigma_{s}\right\}_{s \in S}$ using $k$-means++ (an off-the-shelf vector partitioning algorithm), but this led to highly unbalanced assignments (and thus poor performance). Finally, we note one limitation of our scheme is that if $|S|,|T|=\Theta(n)$, Algorithm 6 essentially partitions the entire graph and thus may be slower than directly estimating PPR. However, we recall from Section 3 that our focus is $|S|,|T|=o(\sqrt{m})=o(n)$, so this is not a concern. Indeed, for the Figure 10 experiment, Algorithm 6 accounted for only $12 \%$ of runtime (averaged across graphs).

\section{CONCLUSION}

In this work, we analyzed the relationship between PPR estimation complexity and clustering by devising estimation algorithms for many node pairs and showing the complexity of these methods scales with quantities interpretable as clustering measures. To demonstrate the utility of these findings, we considered a distributed setting for which the clustering quantities computed in situ could be leveraged to reduce computation time. We believe this setting and the algorithms we designed for it are just one example of how our findings can be used to accelerate PPR estimation; hence, an avenue for future work would be to further explore applications of our results.

\section{APPENDICES}

\section{A ANALYSIS OF FW-BW-MCMC AND COMPARISON TO Bidirectional-PPR}

Here, we state and prove the guarantees that were stated informally at the end of Section 4 . We include the corresponding results for Bidirectional-PPR for comparison. We first present the accuracy guarantee, Theorem A.1. The idea is to bound relative error when $\pi_{s}(t) \geq \delta$ and to bound absolute error when $\pi_{s}(t)<\delta$. The authors of Lofgren et al. [20] suggest choosing $\delta=O\left(\frac{1}{n}\right)$. This choice dictates that we desire the relative bound when $t$ 's PPR exceeds a uniform distribution over all nodes, which suggests $t$ is "significant" to $s$ in this case. The proof applies the Chernoff bound 
to a variety of cases; this approach is similar [20], but we must address cases that do not arise in that work.

Theorem A.1. Fix minimum PPR threshold $\delta$, relative error tolerance $\epsilon$, and failure probability $p_{\text {fail. }}$ For FW-BW-MCMC, assume the following hold:

$$
\epsilon \in\left(0, \frac{1}{\sqrt{2 e}}\right), \quad w=\frac{c r_{\max }^{s} r_{\max }^{t}}{\delta}, \quad c>\frac{3(2 e)^{1 / 3} \log \left(2 / p_{\text {fail }}\right)}{\epsilon^{7 / 3}} .
$$

For Bidirectional-PPR, assume the following hold:

$$
r_{\max }^{t} \in\left(\frac{2 e \delta}{\alpha \epsilon}, 1\right), \quad w=\frac{c r_{\max }^{t}}{\delta}, \quad c>\frac{3 \log \left(2 / p_{\text {fail }}\right)}{\epsilon^{2}} .
$$

Then the estimate $\hat{\pi}_{s}(t)$ produced by either algorithm satisfies the following with probability $\geq 1-$ $p_{\text {fail }}$ :

$$
\left|\pi_{s}(t)-\hat{\pi}_{s}(t)\right| \leq\left\{\begin{array}{ll}
\epsilon \pi_{s}(t), & \pi_{s}(t) \geq \delta \text { ( significant case) } \\
2 e \delta, & \pi_{s}(t)<\delta \text { (insignificant case) }
\end{array} .\right.
$$

Proof. See Lofgren et al. [20] for Bidirectional-PPR; see Appendix B for FW-BW-MCMC.

From Theorem A.1, FW-BW-MCMC offers the same accuracy as Bidirectional-PPR. However, our assumptions on $\epsilon$ and $c$ are stronger than those required for Bidirectional-PPR. The first assumption is mild, since $\frac{1}{\sqrt{2 e}} \approx 0.43$ and we typically desire a tighter relative error bound. The second affects complexity and will be discussed next. Note also our guarantee holds for any $r_{\max }^{t} \in$ $(0,1)$, whereas proving the theorem for Bidirectional-PPR requires a lower bound on $r_{\max }^{t}$.

Next, we have a worst-case complexity result in Theorem A.2 (by worst case, we mean the result holds for when the algorithm is run for any $s, t \in V)$. The idea is to choose $r_{\max }^{s}, r_{\max }^{t}$ to balance the complexity of the DP and MCMC stages of the algorithm. The result requires the additional assumption $m \delta<\log \left(1 / p_{\text {fail }}\right) / \epsilon^{2}$, which guarantees these $r_{\max }^{s}, r_{\max }^{t}$ values lie in $(0,1)$. Note with $\delta=O\left(\frac{1}{n}\right)$, this implies $m=O(n)$ (i.e., nodes have constant degrees as $n$ grows).

Theorem A.2. Fix minimum PPR threshold $\delta$, relative error tolerance $\epsilon$, and failure probability $p_{\text {fail }}$. Assume (27) and (28) hold and $m \delta<\log \left(1 / p_{\text {fail }}\right) / \epsilon^{2}$. Then setting $r_{\max }^{s}=r_{\max }^{t}=\frac{m^{1 / 3} \delta^{1 / 3} \epsilon^{7 / 9}}{\left(\log \left(1 / p_{\text {fail }}\right)\right)^{1 / 3}}$ in FW-BW-MCMC yields minimal complexity $O\left(\frac{m^{2 / 3}\left(\log \left(1 / p_{\text {fail }}\right)\right)^{1 / 3}}{\alpha \epsilon^{7 / 9} \delta^{1 / 3}}\right)$. Furthermore, setting $r_{\max }^{t}=\frac{\sqrt{m \delta} \epsilon}{\sqrt{\log \left(1 / p_{\text {fail }}\right)}}$ in Bidirectional-PPR yields minimal complexity $O\left(\frac{\sqrt{m \log \left(1 / p_{\text {fail }}\right)}}{\alpha \epsilon \sqrt{\delta}}\right)$.

Proof. See Appendix C.

Note with $\delta=O\left(\frac{1}{n}\right)$ so that $m=O(n)$, both algorithms have complexity linear in $n$, whereas FW-BW-MCMC has strictly better dependence on the parameters $p_{\text {fail }}$ and $\epsilon$.

Finally, we present an average-case complexity result for FW-BW-MCMC-Practical (see Algorithm 8), which changes the termination criteria to $\left\|D^{-1} r^{s}\right\|_{\infty} \leq r_{\max }^{s}$ in the forward DP.

Theorem A.3. For any $s \in V$ and for $t \sim V$ uniformly, FW-BW-MCMC-Practical produces an estimate satisfying the accuracy guarantee of Theorem A.1 and has complexity $O\left(\frac{\sqrt{m \log \left(1 / p_{\text {fail }}\right)}}{\sqrt{n \delta} \alpha \epsilon^{7 / 6}}\right)$.

Proof. See Appendix D.

With $\delta=O\left(\frac{1}{n}\right)$, this establishes the $O(\sqrt{m})$ average case complexity claimed at the end of Section 4. The guarantee for Bidirectional-PPR in [20] has $\epsilon$ instead of $\epsilon^{7 / 6}$ but is otherwise identical. 


\section{B PROOF OF THEOREM A.1}

We will use the following result from Dubhashi and Panconesi [8].

Theorem B.1 (From Theorem 1.1 in Dubhashi and Panconesi [8]). Let $\left\{Z_{i}\right\}$ be a set of independent random variables with $Z_{i} \in[0,1] \forall i$, and let $Z=\sum_{i} Z_{i}$. Then for any $\eta \in(0,1)$ and any $d>2 e \mathbb{E}[Z]$,

$$
\begin{gathered}
\mathbb{P}[Z>(1+\eta) \mathbb{E}[Z]] \leq \exp \left(-\eta^{2} \mathbb{E}[Z] / 3\right), \quad \mathbb{P}[Z<(1-\eta) \mathbb{E}[Z]] \leq \exp \left(-\eta^{2} \mathbb{E}[Z] / 2\right), \\
\mathbb{P}[Z>d] \leq 2^{-d} .
\end{gathered}
$$

To begin the proof, we define $Y_{i}=X_{i} / r_{\max }^{t}$ and $Y=\sum_{i=1}^{w} Y_{i}$, where $X_{i}$ is from Algorithm 3 . Observe the $Y_{i}$ 's are independent and $Y_{i} \in[0,1]$ (by the terminating condition of Algorithm 2), so Theorem B.1 applies for appropriate choices of $\eta$ and $d$. We also observe (32) holds, which follows by linearity and $w=\frac{c r_{\max }^{s} r_{\max }^{t}}{\delta}$ in the statement of the theorem.

$$
\mathbb{E}[Y]=\frac{w}{r_{\max }^{t}} \mathbb{E}\left[X_{i}\right]=\frac{c r_{\max }^{s}}{\delta} \mathbb{E}\left[X_{i}\right]
$$

We now turn to the case $\pi_{s}(t) \geq \delta$, for which we aim to show $\mathbb{P}\left[\left|\hat{\pi}_{s}(t)-\pi_{s}(t)\right|>\epsilon \pi_{s}(t)\right]<$ $p_{\text {fail }} \forall \epsilon \in\left(0, \frac{1}{\sqrt{2 e}}\right)$. We will examine three subcases. The first two subcases depend on the constant $k:=\left(\frac{\epsilon}{2 e}\right)^{1 / 3}$ (we motivate the choice of this constant at the conclusion of the proof). We also observe the following, which follows from the assumption $c>\frac{3(2 e)^{1 / 3} \log \left(2 / p_{\text {fail }}\right)}{\epsilon^{7 / 3}}$ :

$$
\frac{k}{3}=\frac{\epsilon}{6 e k^{2}}=\frac{\epsilon^{1 / 3}}{3(2 e)^{1 / 3}}>\frac{\log \left(2 / p_{\text {fail }}\right)}{\epsilon^{2} c} .
$$

For the first subcase, assume $\mathbb{E}[Y] \geq k c$. Then we have the following:

$$
\begin{aligned}
& \mathbb{P}\left[\left|\hat{\pi}_{s}(t)-\pi_{s}(t)\right|>\epsilon \pi_{s}(t)\right] \leq \mathbb{P}\left[\left|\frac{1}{w} \sum_{i=1}^{w} X_{i}-\mathbb{E}\left[X_{i}\right]\right|>\epsilon \mathbb{E}\left[X_{i}\right]\right] \\
& \quad=\mathbb{P}[|Y-\mathbb{E}[Y]|>\epsilon \mathbb{E}[Y]] \leq 2 \exp \left(-\epsilon^{2} \mathbb{E}[Y] / 3\right) \leq 2 \exp \left(-\epsilon^{2} k c / 3\right)<p_{\text {fail }} .
\end{aligned}
$$

Here, the first inequality holds by definition of $\hat{\pi}_{s}(t)$ in Algorithm 3 and the invariant (7); the equality holds by (32) and the definition of $Y$; the second inequality uses Theorem B.1 (note $\epsilon<$ $\frac{1}{\sqrt{2 e}}<1$, so (30) applies); and the final two inequalities hold by $\mathbb{E}[Y] \geq k c$ and (33), respectively.

For the second subcase, assume $\mathbb{E}[Y] \in\left[\frac{\epsilon c}{2 e}, k c\right)$. First, observe that by (32), the assumption $\mathbb{E}[Y]<k c$, and the Algorithm 1 terminating condition,

$$
\left\|r^{s}\right\|_{1} \mathbb{E}\left[X_{i}\right]=\frac{\left\|r^{s}\right\|_{1} \delta \mathbb{E}[Y]}{c r_{\max }^{s}}<\frac{\left\|r^{s}\right\|_{1} k \delta}{r_{\max }^{s}} \leq k \delta,
$$

and so $\pi_{s}(t) \geq\left\|r^{s}\right\|_{1} \mathbb{E}\left[X_{i}\right]+(1-k) \delta$ (else, $\pi_{s}(t)<\delta$ by (7), a contradiction). We then write the following:

$$
\begin{aligned}
\mathbb{P} & {\left[\left|\hat{\pi}_{s}(t)-\pi_{s}(t)\right|>\epsilon \pi_{s}(t)\right] \leq \mathbb{P}\left[\left|\frac{1}{w} \sum_{i=1}^{w} X_{i}-\mathbb{E}\left[X_{i}\right]\right|>\epsilon\left(\mathbb{E}[X]+\frac{(1-k) \delta}{\left\|r^{s}\right\|_{1}}\right)\right] } \\
& =\mathbb{P}\left[|Y-\mathbb{E}[Y]|>\epsilon\left(\mathbb{E}[Y]+\frac{(1-k) \delta w}{\left\|r^{s}\right\|_{1} r_{\text {max }}^{t}}\right)\right] \leq \mathbb{P}[|Y-\mathbb{E}[Y]|>\epsilon(\mathbb{E}[Y]+(1-k) c)] \\
& =\mathbb{P}\left[|Y-\mathbb{E}[Y]|>\epsilon\left(\mathbb{E}[Y]+\left(\frac{1-k}{k}\right) k c\right)\right]<\mathbb{P}\left[|Y-\mathbb{E}[Y]|>\frac{\epsilon}{k} \mathbb{E}[Y]\right] \\
& \leq 2 \exp \left(-\epsilon^{2} \mathbb{E}[Y] /\left(3 k^{2}\right)\right)<2 \exp \left(-\epsilon^{3} c /\left(6 e k^{2}\right)\right)<p_{\text {fail }} .
\end{aligned}
$$


Here, the first inequality and first equality follow similar arguments as Case 1; the second inequality is by the Algorithm 1 terminating condition and $w=\frac{c r_{\max }^{s} r_{\max }^{t}}{\delta}$; the second equality simply multiplies and divides $k$; the third inequality holds by assumption $\mathbb{E}[Y] \in\left[\frac{\epsilon c}{2 e}, k c\right)$; the fourth inequality holds by Theorem B.1 (note $\frac{\epsilon}{k}=\epsilon^{2 / 3}(2 e)^{1 / 3}<1$ by assumption $\epsilon<\frac{1}{\sqrt{2 e}}$, so (30) applies); the fifth inequality follows from $\mathbb{E}[Y] \in\left[\frac{\epsilon c}{2 e}, k c\right)$; and the final inequality holds by (33). Note we have assumed $1-k>0$ in the third and fifth inequality; this follows from $\epsilon<\frac{1}{\sqrt{2 e}}$.

For the third and final subcase, assume $\mathbb{E}[Y]<\frac{\epsilon c}{2 e}$. We have the following:

$$
\begin{aligned}
& \mathbb{P}\left[\left|\hat{\pi}_{s}(t)-\pi_{s}(t)\right|>\epsilon \pi_{s}(t)\right]=\mathbb{P}\left[\left|\frac{1}{w} \sum_{i=1}^{w} X_{i}-\mathbb{E}\left[X_{i}\right]\right|>\frac{\epsilon \pi_{s}(t)}{\left\|r^{s}\right\|_{1}}\right]=\mathbb{P}\left[|Y-\mathbb{E}[Y]|>\frac{\epsilon \pi_{s}(t) w}{\left\|r^{s}\right\|_{1} r_{\text {max }}^{t}}\right] \\
& \leq \mathbb{P}\left[|Y-\mathbb{E}[Y]|>\frac{\epsilon \delta w}{r_{\text {max }}^{s} r_{\text {max }}^{t}}\right]=\mathbb{P}[|Y-\mathbb{E}[Y]|>\epsilon c] \leq \mathbb{P}[Y>\epsilon c] \leq 2^{-\epsilon c} .
\end{aligned}
$$

Here, the first three equalities and first inequality follow similar arguments as previous cases; the penultimate inequality holds since $\{|Y-\mathbb{E}[Y]|>\epsilon c\} \subset\{Y>\epsilon c\}$ when $Y \geq \mathbb{E}[Y]$, whereas $\{\mid Y-$ $\mathbb{E}[Y] \mid>\epsilon c\} \subset\{\mathbb{E}[Y]>\epsilon c\} \subset\{2 e \mathbb{E}[Y]>\epsilon c\}=\emptyset$ when $Y<\mathbb{E}[Y]$; and the final inequality holds by Theorem B.1; note $\epsilon c>2 e \mathbb{E}[Y]$ by assumption, so (31) applies. Next, we observe

$$
\epsilon c>\frac{6 e \log \left(1 / p_{\text {fail }}\right)}{(\sqrt{2 e} \epsilon)^{4 / 3}}>6 e \log \left(1 / p_{\text {fail }}\right)=\frac{6 e}{\log _{2}(e)} \log _{2}\left(1 / p_{\text {fail }}\right)>\log _{2}\left(1 / p_{\text {fail }}\right),
$$

where the first two inequalities hold by $c>\frac{3(2 e)^{1 / 3} \log \left(1 / p_{\text {fail }}\right)}{\epsilon^{7 / 3}}$ and $\epsilon<\frac{1}{\sqrt{2 e}}$, and the final inequality holds since $e<4 \Rightarrow \log _{2}(e)<2 \Rightarrow \frac{6 e}{\log _{2}(e)}>\frac{3 e}{2}>1$. Combining (34) and (35) completes Case 3 .

Finally, we note the bounds in Cases 1 and 3 grow with decreasing and increasing $k$, respectively. Hence, our choice of $k=\left(\frac{\epsilon}{2 e}\right)^{1 / 3}$ comes from equating the two to minimize failure probability.

We now turn to the case $\pi_{s}(t)<\delta$. Observe that by $\pi_{s}(t)<\delta$ and the invariant $(7),\left\|r^{s}\right\|_{1} \mathbb{E}\left[X_{i}\right]<$ $\delta$. By (32), this implies $2 e \mathbb{E}[Y]<\frac{2 e w \delta}{r_{\max }^{t}\left\|r^{s}\right\|_{1}}=: b$. Then

$$
\begin{aligned}
\mathbb{P}\left[\left|\hat{\pi}_{s}(t)-\pi_{s}(t)\right|>2 e \delta\right] & =\mathbb{P}\left[\left|\frac{1}{w} \sum_{i=1}^{w} X_{i}-\mathbb{E}\left[X_{i}\right]\right|>\frac{2 e \delta}{\left\|r^{s}\right\|_{1}}\right]=\mathbb{P}\left[|Y-\mathbb{E}[Y]|>\frac{2 e \delta w}{\left\|r^{s}\right\|_{1} r_{\max }^{t}}\right] \\
& =\mathbb{P}[|Y-\mathbb{E}[Y]|>b] \leq P[Y>b] \leq 2^{-b} .
\end{aligned}
$$

Here, the equalities follow similar steps as previous cases, the first inequality holds by the same argument in the Case 3 analysis, and the final inequality holds by Theorem B.1 (note (31) applies since $b>2 e \mathbb{E}[Y])$. We also observe

$$
b=\frac{2 e w \delta}{r_{\max }^{t}\left\|r^{s}\right\|_{1}}>\frac{2 e w \delta}{r_{\max }^{t} r_{\max }^{s}}=2 e c>\epsilon c>\log _{2}\left(1 / p_{\text {fail }}\right),
$$

where the first inequality is by the Algorithm 1 terminating condition, the second inequality holds since $2 e>1>\epsilon$, and the third inequality follows from (35); the equalities are by definition. Finally, we combine (36) and (37) to complete the proof.

\section{PROOF OF THEOREM A.2}

The complexity of Algorithm 3 is the total complexity of Algorithm 2, Algorithm 1, and the random walks. In the following, we show Algorithms 2 and 1 have complexity $\frac{m}{\alpha r_{\max }^{t}}$ and $\frac{m}{\alpha r_{\max }^{s}}$, respectively (using arguments from Andersen et al. [1,2]). Furthermore, the complexity of the random walk stage is $O\left(\frac{r_{\max }^{s} r_{\max }^{t} \log \left(1 / p_{\text {fail }}\right)}{\alpha \delta \epsilon^{7 / 3}}\right)$, where $\frac{1}{\alpha}$ is the expected complexity of sampling a single random walk, 
and where the remaining factors give the number of walks required (recall in the statement of the theorem we assume (27) holds). Hence, the complexity of Algorithm 3 is $O\left(C\left(r_{\max }^{s} r_{\max }^{t}\right) / \alpha\right)$, where

$$
C\left(r_{\max }^{s} r_{\max }^{t}\right)=\frac{m}{r_{\max }^{s}}+\frac{r_{\max }^{s} r_{\max }^{t} \log \left(1 / p_{\text {fail }}\right)}{\delta \epsilon^{7 / 3}}+\frac{m}{r_{\max }^{t}}
$$

We now aim choose $r_{\max }^{s}, r_{\max }^{t}$ to minimize $O\left(C\left(r_{\max }^{s} r_{\max }^{t}\right) / \alpha\right)$, or equivalently, to minimize $C\left(r_{\max }^{s} r_{\max }^{t}\right)$. For this, we let $K=\frac{\log \left(1 / p_{\text {fail }}\right)}{\delta \epsilon^{7 / 3}}>0$ and note $\frac{\partial C}{\partial r_{\max }^{s}}=K r_{\max }^{t}-\frac{m}{\left(r_{\max }^{s}\right)^{2}}=0$ if and only if $\left(r_{\max }^{s}\right)^{2} r_{\max }^{t}=\frac{m}{K}$, and similarly, $\frac{\partial C}{\partial r_{\max }^{t}}=0$ if and only if $\left(r_{\max }^{t}\right)^{2} r_{\max }^{s}=\frac{m}{K}$; hence, $\left(\frac{m}{K}\right)^{1 / 3},\left(\frac{m}{K}\right)^{1 / 3}$ is a stationary point of $C\left(r_{\max }^{s}, r_{\max }^{t}\right)$. To verify this is a minimizer, we observe

$$
\left[\begin{array}{cc}
\frac{\partial^{2} C}{\partial\left(r_{\max }^{s}\right)^{2}} & \frac{\partial^{2} C}{\partial r_{\max }^{s} r_{\max }^{t}} \\
\frac{\partial^{2} C}{\partial r_{\max }^{t} r_{\max }^{s}} & \frac{\partial^{2} C}{\partial\left(r_{\max }^{t}\right)^{2}}
\end{array}\right]=\left[\begin{array}{cc}
\frac{2 m}{\left(r_{\max }^{s}\right)^{3}} & K \\
K & \frac{2 m}{\left(r_{\max }^{t}\right)^{3}}
\end{array}\right]
$$

from which it follows that the Hessian of $C$ evaluated at $r_{\max }^{s}=r_{\max }^{t}=\left(\frac{m}{K}\right)^{1 / 3}$ is $K\left(I+11^{\top}\right)$. This is positive definite, since for any vector $z \neq 0$,

$$
z^{\top}\left(K\left(I+11^{\top}\right)\right) z=K\left(\|z\|_{2}^{2}+\left(z^{\top} 1\right)^{2}\right)>0
$$

To summarize, we have shown $r_{\max }^{s}=r_{\max }^{t}=\left(\frac{m}{K}\right)^{1 / 3}$ minimizes $C\left(r_{\max }^{s}, r_{\max }^{t}\right)$ and hence minimizes the complexity of Algorithm 3. This establishes that the choice of $r_{\max }^{s}, r_{\max }^{t}$ in the statement of the theorem minimizes complexity. Finally, substituting $r_{\max }^{s}=r_{\max }^{t}=\left(\frac{m}{K}\right)^{1 / 3}$ into (38) and dividing by $\alpha$ gives the complexity expression given in the theorem. Following the same approach establishes the Algorithm Bidirectional-PPR complexity bound given in the theorem.

We return to bound the complexities of Algorithms 2 and 1. For Algorithm 2, we use an argument from Andersen et al. [1]. First, let $v \in V$. From Algorithm 2, $p^{t}(v)$ increases by at least $\alpha r_{\max }^{t}$ at each iteration for which $v^{*}=v$. By the invariant (6), $p^{t}(v) \leq \pi_{v}(t)$. Taken together, $v^{*}=v$ for at most $\frac{\pi_{v}(t)}{\alpha r_{\max }^{t}}$ iterations. Furthermore, the complexity of each iteration for which $v^{*}=v$ is $d_{\text {in }}(v)$. Hence, the complexity of all iterations for which $v^{*}=v$ is bounded by $d_{\text {in }}(v) \frac{\pi_{v}(t)}{\alpha r_{\max }^{t}}$. Finally, the complexity of Algorithm 2 can be bounded by summing over all $v \in V$-that is, $\sum_{v \in V} d_{\text {in }}(v) \frac{\pi_{v}(t)}{\alpha r_{\max }^{t}} \leq$ $\frac{1}{\alpha r_{\max }^{t}} \sum_{v \in V} d_{\text {in }}(v)=\frac{m}{\alpha r_{\max }^{t}}$.

We next turn to Algorithm 1. As mentioned in the main text, Algorithm 1 changes the termination criteria from the algorithm originally defined in Andersen et al. [2]; to clarify, we include the original definition in Algorithm 7. Here, we use tilde marks to distinguish quantities from those in Algorithm 1, and we explicitly indicate iteration number $k$ to improve clarity of the arguments to follow. Besides these notational changes, the only difference between Algorithms 1 and 7 is the termination criteria.

With this notation in place, the complexity of Algorithm 7 can be bounded as follows (using arguments from Andersen et al. [2]). First, observe for any iteration $k$,

$$
\left\|\tilde{r}_{k}^{s}\right\|_{1}=\sum_{v \in V \backslash\left(\left\{v_{k}\right\} \cup N_{\text {out }}\left(v_{k}\right)\right)} \tilde{r}_{k-1}^{s}(v)+\sum_{v \in N_{\text {out }}\left(v_{k}\right)}\left(\tilde{r}_{k-1}^{s}(v)+\frac{(1-\alpha) \tilde{r}_{k-1}^{s}\left(v_{k}\right)}{d_{\text {out }}\left(v_{k}\right)}\right)=\left\|\tilde{r}_{k-1}^{s}\right\|_{1}-\alpha \tilde{r}_{k-1}^{s}\left(v_{k}\right),
$$

where the first equality holds via the iterative update in Algorithm 7. Next, let $k^{*}$ be the iteration at which Algorithm 7 terminates. Then the complexity of the algorithm is $\sum_{k=1}^{k^{*}} d_{\text {out }}\left(v_{k}\right)$, and 


$$
\begin{aligned}
\sum_{k=1}^{k^{*}} d_{\text {out }}\left(v_{k}\right) & =\sum_{k=1}^{k^{*}} \frac{d_{\text {out }}\left(v_{k}\right)}{\tilde{r}_{k-1}^{s}\left(v_{k}\right)} \tilde{r}_{k-1}^{s}\left(v_{k}\right)<\frac{1}{\tilde{r}_{\max }^{s}} \sum_{k=1}^{k^{*}} \tilde{r}_{k-1}^{s}\left(v_{k}\right) \\
& =\frac{1}{\alpha \tilde{r}_{\max }^{s}} \sum_{k=1}^{k^{*}}\left(\left\|\tilde{r}_{k-1}^{s}\right\|_{1}-\left\|\tilde{r}_{k}^{s}\right\|_{1}\right)=t \frac{1}{\alpha \tilde{r}_{\max }^{s}}\left(\left\|\tilde{r}_{0}^{s}\right\|_{1}-\left\|\tilde{r}_{k^{*}}^{s}\right\|_{1}\right) \leq \frac{1}{\alpha \tilde{r}_{\max }^{s}},
\end{aligned}
$$

where the first inequality holds since $\tilde{r}_{\max }^{s}<\left\|D^{-1} \tilde{r}_{k}^{s}\right\|_{\infty}=\frac{\tilde{r}_{k-1}^{s}\left(v_{k}\right)}{d_{\text {out }}\left(v_{k}\right)}$ for $k \leq k^{*}$ (i.e., for each $k$ until the algorithm terminates), the second equality holds by the previous display, and the final inequality holds since $\left\|\tilde{r}_{0}^{s}\right\|_{1}=\left\|e_{s}\right\|_{1}=1$ and $\left\|\tilde{r}_{k^{*}}^{s}\right\|_{1} \geq 0$ (the remaining steps are straightforward).

Using this, we can bound the complexity of Algorithm 1. First, observe that in Algorithm 1,

$$
\left\|r^{s}\right\|_{1}=\sum_{v \in V} \frac{r^{s}(v)}{d_{\text {out }}(v)} d_{\text {out }}(v) \leq m \max _{v \in V} \frac{r^{s}(v)}{d_{\text {out }}(v)}=m\left\|D^{-1} r^{s}\right\|_{\infty},
$$

and so to guarantee termination of Algorithm 1 (i.e., to ensure $\left\|r^{s}\right\|_{1} \leq r_{\max }^{s}$ ), it suffices to guarantee $\left\|D^{-1} r^{s}\right\|_{\infty} \leq \frac{r_{\max }^{s}}{m}$. But from the analysis of Algorithm 7 , the complexity required to ensure $\left\|D^{-1} r^{s}\right\|_{\infty} \leq \frac{r_{\max }^{s}}{m}$ is $\frac{m}{\alpha r_{\max }^{s}}$; hence, the complexity of Algorithm 1 is bounded by $\frac{m}{\alpha r_{\max }^{s}}$ as well.

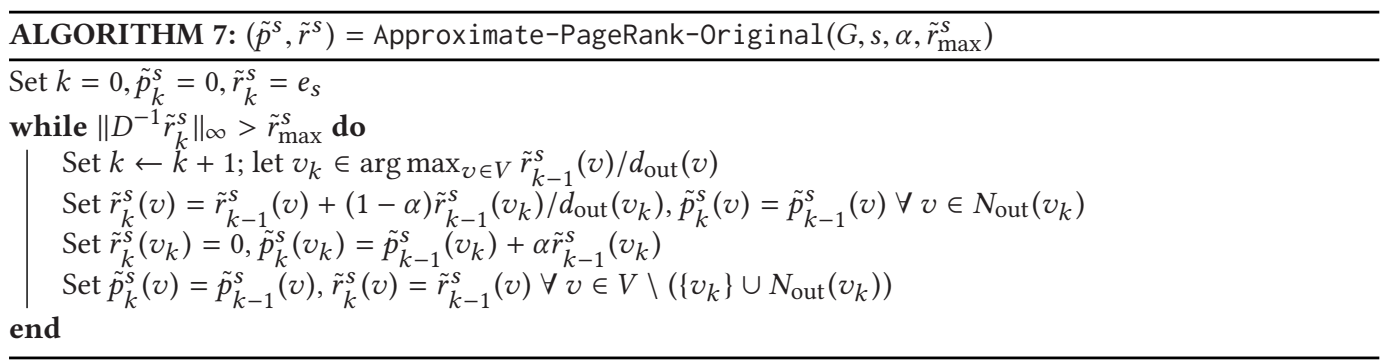

\section{PRACTICAL VERSION OF FW-BW-MCMC}

In this appendix, we define and analyze a modified version of FW-BW-MCMC that is more useful in practice. Before proceeding to the formal definition and analysis, we first motivate the practical algorithm. First, suppose for an instance of FW-BW-MCMC we have already run the backward DP (Algorithm 2) and are currently running the forward DP (Algorithm 1). Although FW-BW-MCMC dictates we run the forward DP until $\left\|r^{s}\right\|_{1}<r_{\text {max }}^{s}$ for some predefined $r_{\text {max }}^{s}$, we could instead terminate the forward DP (even if $\left\|r^{s}\right\|_{1}>r_{\max }^{s}$ ) and proceed to the random walks. In other words, we dynamically change $r_{\max }^{s}$ from the predefined value to the current value of $\left\|r^{s}\right\|_{1}$. Then, if the number of walks sampled is $w=c\left\|r^{s}\right\|_{1} r_{\max }^{t} / \delta$, where

$$
c=\frac{3(2 e)^{1 / 3} \log \left(2 / p_{\mathrm{fail}}\right)}{\epsilon^{7 / 3}},
$$

the proof of Theorem A.1 goes through (i.e., the accuracy guarantee is achieved). Furthermore, this argument holds at any iteration of the forward DP. In other words, we can terminate the forward DP at any iteration and achieve the accuracy guarantee, as long as we scale $w$ with the $\left\|r^{s}\right\|_{1}$ value obtained at termination. From this observation, we aim to terminate the forward DP at the "optimal" iteration-that is, the iteration for which the overall complexity of the algorithm is minimized.

Toward determining this optimal iteration, let $C_{F D P}$ denote the complexity of the forward DP until the current iteration, and define $C_{M C M C}=\frac{3(2 e)^{1 / 3} r_{\max }^{t} \log \left(2 / p_{\text {fail }}\right)}{\alpha \delta \epsilon^{7 / 3}}$, so that $\left\|r^{s}\right\|_{1} C_{M C M C}$ gives the 
complexity of the MCMC stage (since $c\left\|r^{s}\right\|_{1} r_{\max }^{t} / \delta$ walks are sampled, each in expected time $\frac{1}{\alpha}$, with $c$ satisfying (40)). Then, if we terminate the forward DP at the current iteration, the combined complexity of forward DP and MCMC stages will be $C_{F D P}+\left\|r^{s}\right\|_{1} C_{M C M C}$. Suppose instead we decide to run one more iteration (i.e., to terminate the forward DP at the next iteration). Then, by Algorithm 1, the next iteration will have complexity $d_{\text {out }}\left(v^{*}\right)$. Furthermore, by (39) in Appendix C, $\left\|r^{s}\right\|_{1}$ will decrease by $\alpha r^{s}\left(v^{*}\right)$ at the next iteration. Hence, if we run one more iteration, the combined complexity of forward DP and MCMC will be $\left(C_{F D P}+d_{\text {out }}\left(v^{*}\right)\right)+\left(\left\|r^{s}\right\|_{1}-\alpha r^{s}\left(v^{*}\right)\right) C_{M C M C}$. Now clearly, we should terminate the forward DP if and only if the resulting complexity is less than the complexity resulting from running another iteration. Hence, from the previous argument, we should terminate if and only if

$$
C_{F D P}+\left\|r^{s}\right\|_{1} C_{M C M C}<\left(C_{F D P}+d_{\text {out }}\left(v^{*}\right)\right)+\left(\left\|r^{s}\right\|_{1}-\alpha r^{s}\left(v^{*}\right)\right) C_{M C M C} \Leftrightarrow \frac{r^{s}\left(v^{*}\right)}{d_{\text {out }}\left(v^{*}\right)}<\frac{1}{\alpha C_{M C M C}} .
$$

In other words, to optimize the tradeoff between forward DP and MCMC complexity, we should run the forward DP until $\left\|D^{-1} r^{s}\right\|_{\infty}$ falls below the threshold in (41). This motivates the practical version of FW-BW-MCMC, given in Algorithm 8. Algorithm 8 changes two aspects of FW-BW-MCMC. First, it replaces Algorithm 1 with Algorithm 7 (which uses $\left\|D^{-1} \tilde{r}^{s}\right\|_{\infty}$ termination, as suggested by (41)). Second, it scales the the number of random walks sampled with $\left\|\tilde{r}^{s}\right\|_{1}$, as discussed earlier.

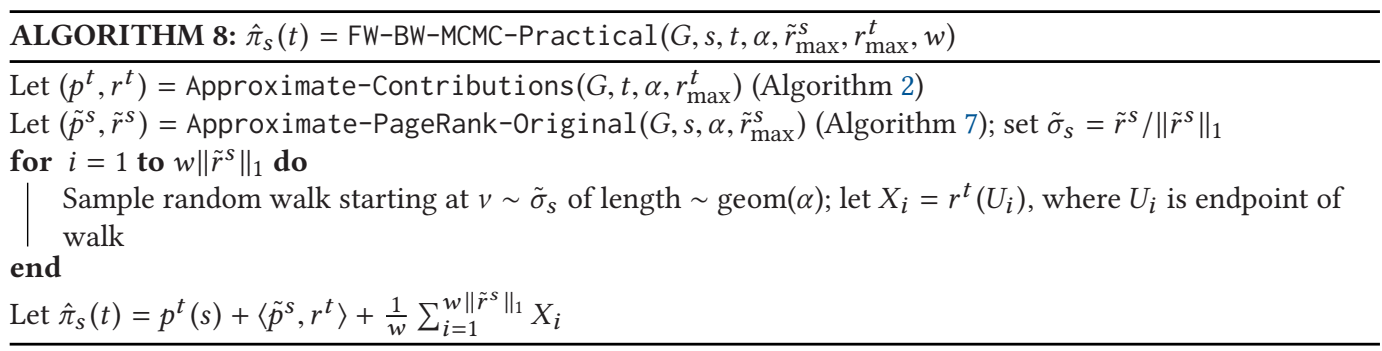

We can now establish accuracy and average-case complexity guarantees for Algorithm 8.

Theorem D.1. Fix minimum PPR threshold $\delta$, relative error tolerance $\epsilon$, and failure probability $p_{\text {fail. }}$ Let

$$
\epsilon \in\left(0, \frac{1}{\sqrt{2 e}}\right), \quad w=\frac{c r_{\max }^{t}}{\delta}, \quad c>\frac{3(2 e)^{1 / 3} \log \left(2 / p_{\text {fail }}\right)}{\epsilon^{7 / 3}} .
$$

Then the estimate $\hat{\pi}_{s}(t)$ produced by Algorithm 8 satisfies (29)with probability $\geq 1-p_{\text {fail }}$.

Proof. As discussed earlier, the proof of Theorem A.1 goes through to establish this result.

Theorem D.2. Fix minimum PPR threshold $\delta$, relative error tolerance $\epsilon$, and failure probability $p_{\text {fail }}$. Assume (42) holds. Then for any $s \in V$ and for $t \sim V$ uniformly, setting $\tilde{r}_{\max }^{s}=\frac{\delta \epsilon^{7 / 3}}{r_{\max }^{t} \log \left(1 / p_{\text {fail }}\right)}$, $r_{\max }^{t}=\frac{\sqrt{m \delta} \epsilon^{7 / 6}}{\sqrt{n \log \left(1 / p_{\text {fail }}\right)}}$ in Algorithm 8 yields complexity $O\left(\frac{\sqrt{m \log \left(1 / p_{\text {fail }}\right)}}{\sqrt{n \delta} \alpha \epsilon^{7 / 6}}\right)$.

Proof. First, for the complexity of the backward DP (Algorithm 2), we use the result from Lofgren and Goel [21], which we include for completeness. Recall from Appendix C that the complexity of Algorithm 2 for any $t \in V$ is bounded by $\sum_{v \in V} d_{\text {in }}(v) \frac{\pi_{v}(t)}{\alpha r_{\max }^{t}}$. Hence, for $t \sim V$ uniformly, 
the expected complexity is

$$
\frac{1}{n} \sum_{t \in V} \sum_{v \in V} d_{\text {in }}(v) \frac{\pi_{v}(t)}{\alpha r_{\max }^{t}}=\frac{1}{n \alpha r_{\max }^{t}} \sum_{v \in V} d_{\text {in }}(v) \sum_{t \in V} \pi_{v}(t)=\frac{m}{n \alpha r_{\max }^{t}},
$$

since $\sum_{t \in V} \pi_{v}(t)=1$ by definition. Next, we consider the complexity of the forward DP (Algorithm 7). From Appendix C, for any $s \in V$, we have complexity $\frac{1}{\alpha \tilde{r}_{\max }^{s}}=\frac{r_{\max }^{t} \log \left(1 / p_{\text {fail }}\right)}{\alpha \delta \epsilon^{7 / 3}}$. Finally, for the MCMC stage, we sample $w\left\|\tilde{r}^{s}\right\|_{1} \leq w$ walks, where $w=c r_{\max }^{t} / \delta$ with $c$ satisfying (42). Each walk is sampled in average time $\frac{1}{\alpha}$. Therefore, the MCMC stage complexity is $O\left(\frac{r_{\max }^{t} \log \left(1 / p_{\text {fail }}\right)}{\alpha \delta \epsilon^{7 / 3}}\right)$. Thus, the overall complexity of Algorithm 8 is bounded by

$$
O\left(\frac{m}{n \alpha r_{\max }^{t}}+\frac{r_{\max }^{t} \log \left(1 / p_{\mathrm{fail}}\right)}{\alpha \delta \epsilon^{7 / 3}}\right)
$$

Substituting $r_{\text {max }}^{t}$ given in the statement of the theorem yields the desired complexity bound. Further, viewing (43) as a function of $r_{\max }^{t}$, it is straightforward to verify this $r_{\max }^{t}$ is the global minimizer.

\section{E PROOF OF THEOREM 5.1}

We first observe

$$
\begin{aligned}
& \mathbb{P}\left[\sum_{v \in V} \max _{s \in S} X_{s}^{(w)}(v)>(1+\epsilon) w \sum_{v \in V} \max _{s \in S} \sigma_{s}(v)\right] \\
& \leq \mathbb{P}\left[\cup_{s \in S, v \in V}\left\{X_{s}^{(w)}(v)>(1+\epsilon) w \sigma_{s}(v)\right\}\right] \leq \sum_{s \in S, v \in V: \sigma_{s}(v)>0} \mathbb{P}\left[X_{s}^{(w)}(v)>(1+\epsilon) w \sigma_{s}(v)\right],
\end{aligned}
$$

where the second inequality uses the fact that $X_{s}^{(w)}(v) \sim \operatorname{Binomial}\left(w, \sigma_{s}(v)\right)$ (hence, $X_{s}^{(w)}(v)=0$ when $\sigma_{s}(v)=0$ ). Again using this fact, we have by (30) from Theorem B.1 in Appendix B,

$$
\mathbb{P}\left[X_{s}^{(w)}(v)>(1+\epsilon) w \sigma_{s}(v)\right] \leq \exp \left(-\frac{\epsilon^{2}}{3} w \sigma_{s}(v)\right) .
$$

Combining (44) and (45), we obtain

$$
\begin{aligned}
\mathbb{P} & {\left[\sum_{v \in V} \max _{s \in S} X_{s}^{(w)}(v)>(1+\epsilon) w \sum_{v \in V} \max _{s \in S} \sigma_{s}(v)\right] \leq \sum_{s \in S, v \in V: \sigma_{s}(v)>0} \exp \left(-\frac{\epsilon^{2}}{3} w \sigma_{s}(v)\right) } \\
& \left.\leq \max _{s \in S, v \in V: \sigma_{s}(v)>0}\left\{\exp \left(-\frac{\epsilon^{2}}{3} w \sigma_{s}(v)\right)\right\}\right)\left(\sum_{s \in S, v \in V} \mathbb{1}_{\left\{\sigma_{s}(v)>0\right\}}\right) \\
& =\exp \left(\frac{-\epsilon^{2}}{3} w \min _{s \in S, v \in V: \sigma_{s}(v)>0} \sigma_{S}(v)\right)\left(\sum_{s \in S, v \in V} \mathbb{1}_{\left\{\sigma_{s}(v)>0\right\}}\right)<p_{\text {fail }} / 2,
\end{aligned}
$$

where the final inequality holds by the bound on $w$ in the statement of the theorem. For the lower tail, following the same steps used to obtain (46) gives

$$
\mathbb{P}\left[\sum_{v \in V} \max _{s \in S} X_{s}^{(w)}(v)<(1-\epsilon) w \sum_{v \in V} \max _{s \in S} \sigma_{s}(v)\right]<p_{\text {fail }} / 2 .
$$

Finally, by the union bound, (46) and (47) together establish the theorem. 


\section{F PROOF OF PROPOSITION 5.3}

First, assume Merge is used at each iteration for which $v^{*}=t_{2}$. By Algorithm 2, $\left\|p^{t_{2}}\right\|_{1}$ increases by at least $\alpha r_{\max }^{t}$ at each iteration for which $v^{*} \neq t_{1}$. By (13), $\left\|p^{t_{2}}\right\|_{1}$ increases by at least $\left\|p^{t_{1}}\right\|_{1} r_{\max }^{t}$ at each iteration for which $v^{*}=t_{1}$. Let us define $I_{1}$ as the number of iterations for which $v^{*} \neq t_{1}$, $I_{2}$ as the number of iterations for which $v^{*}=t_{1}$, and $I=I_{1}+I_{2}$ as the total number of iterations. Since $\left\|p^{t_{2}}\right\|_{1}=0$ at the start of Algorithm 2 and $\left\|p^{t_{2}}\right\|_{1} \leq n \pi\left(t_{2}\right)$ by the invariant (6), we have

$$
\frac{n \pi\left(t_{2}\right)}{r_{\max }^{t}} \geq \alpha I_{1}+\left\|p^{t_{1}}\right\|_{1} I_{2}=\alpha I+\left(\left\|p^{t_{1}}\right\|_{1}-\alpha\right) I_{2}
$$

Now at termination of Algorithm 2, $\left\|r^{t_{2}}\right\|_{\infty} \leq r_{\max }^{t}$, so by the invariant (6), $\pi_{t_{1}}\left(t_{2}\right) \leq p^{t_{2}}\left(t_{1}\right)+r_{\max }^{t}$ at termination. Therefore, if $\pi_{t_{1}}\left(t_{2}\right)>r_{\max }^{t}, p^{t_{2}}\left(t_{1}\right)>0$ at termination, which can only occur if $v^{*}=t_{1}$ at some iteration. Hence, $\pi_{t_{1}}\left(t_{2}\right)>r_{\max }^{t} \Rightarrow I_{2} \geq 1$. Finally, from Algorithm 2, $\left\|p^{t_{1}}\right\|_{1} \geq \alpha$. Substituting into (48) gives $I \leq \frac{n \pi\left(t_{2}\right)}{\alpha r_{\max }^{t}}-\frac{\left(\left\|p^{t_{1}}\right\|_{1}-\alpha\right)}{\alpha}$.

If instead Merge is not used, $\left\|p^{t_{2}}\right\|_{1}$ increases by at least $\alpha r_{\max }^{t}$ at every iteration. Hence, the same argument as earlier establishes the total number of iterations is bounded by $\frac{n \pi\left(t_{2}\right)}{\alpha r_{\max }^{t}}$.

\section{G PROOF OF THEOREM 5.4}

We will use Corollary 6.2.1 from Tropp [28], which (applied to our setting) states the following. Assume $\left\{X_{i}\right\}_{i=1}^{w}$ are independent random matrices satisfying $\mathbb{E}\left[X_{i}\right]=R_{S}^{\mathrm{T}} \Pi R_{T}$. Let $M$ be subject to $\left\|X_{i}\right\|_{2} \leq M$ almost surely, and let $m_{2}\left(X_{i}\right)=\max \left\{\left\|\mathbb{E}\left[X_{i} X_{i}^{\top}\right]\right\|_{2},\left\|\mathbb{E}\left[X_{i}^{\top} X_{i}\right]\right\|_{2}\right\}$. Then $\forall \eta>0$,

$$
\mathbb{P}\left[\left\|R_{S}^{\top} \Pi R_{t}-\frac{1}{w} \sum_{i=1}^{w} X_{i}\right\|_{2}>\eta\right] \leq 2 l \exp \left(\frac{-3 w \eta^{2}}{6 m_{2}\left(X_{i}\right)+4 M \eta}\right) .
$$

We have verified the independence and $\mathbb{E}\left[X_{i}\right]=R_{S}^{\mathrm{T}} \Pi R_{T}$ assumptions in the main text. Furthermore, from (19) and Algorithm 5, $\Pi(S, T)-\hat{\Pi}(S, T)=R_{S}^{\top} \Pi R_{t}-\frac{1}{w} \sum_{i=1}^{w} X_{i}$. We may therefore write

$$
\begin{aligned}
& \mathbb{P}\left[\|\Pi(S, T)-\hat{\Pi}(S, T)\|_{2}>\epsilon \max \left\{\|\Pi(S, T)\|_{2}, 1\right\}\right] \leq 2 l \exp \left(\frac{-3 w\left(\epsilon \max \left\{\|\Pi(S, T)\|_{2}, 1\right\}\right)^{2}}{6 m_{2}\left(X_{i}\right)+4 M \epsilon \max \left\{\|\Pi(S, T)\|_{2}, 1\right\}}\right) \\
& \quad \leq 2 l \exp \left(\frac{-3 w \epsilon^{2}}{6 \frac{m_{2}\left(X_{i}\right)}{\max \left\{\|\Pi(S, T)\|_{2}, 1\right\}}+4 M \epsilon}\right) \leq 2 l \exp \left(\frac{-3 w \epsilon^{2}}{6 \frac{m_{2}\left(X_{i}\right)}{\|\Pi(S, T)\|_{2}}+4 M \epsilon}\right),
\end{aligned}
$$

where we have also used the inequalities $\max \left\{\|\Pi(S, T)\|_{2}, 1\right\} \geq 1, \max \left\{\|\Pi(S, T)\|_{2}, 1\right\} \geq\|\Pi(S, T)\|_{2}$.

Now to prove the theorem, we aim to find $M$ subject to $\left\|X_{i}\right\|_{2} \leq M$ almost surely and to compute $m_{2}\left(X_{i}\right)$ such that (49) is bounded by $p_{\text {fail }}$, in each of the following cases:

$$
\begin{gathered}
\text { (Case 1) } \sigma=\sigma_{\mathrm{avg}}, \quad w \geq \frac{l^{2} \sqrt{\operatorname{srank}(\Pi(S, T))} \log \left(2 l / p_{\mathrm{fail}}\right) r_{\max }^{s} r_{\max }^{t}(6+4 \epsilon)}{3 \epsilon^{2}}, \\
\text { (Case 2) } \sigma=\sigma_{\max }, \quad w \geq \frac{l^{3 / 2}\|\Sigma\|_{\infty, 1} \log \left(2 l / p_{\mathrm{fail}}\right) r_{\max }^{s} r_{\max }^{t}(6+4 \epsilon)}{3 \epsilon^{2}} .
\end{gathered}
$$

We begin with Case 1. By Lemma G.1, we may take $M=l^{3 / 2} r_{\max }^{s} r_{\max }^{t}$, and by Lemma G.2, we have $m_{2}\left(X_{i}\right) \leq l^{2} r_{\max }^{s} r_{\max }^{t}\|\Pi(S, T)\|_{F}$. We can then write

$$
\begin{aligned}
6 \frac{m_{2}\left(X_{i}\right)}{\|\Pi(S, T)\|_{2}}+4 M \epsilon & \leq l^{2} r_{\max }^{s} r_{\max }^{t}\left(6 \frac{\|\Pi(S, T)\|_{F}}{\|\Pi(S, T)\|_{2}}+\frac{4}{\sqrt{l}} \epsilon\right)=l^{2} r_{\max }^{s} r_{\max }^{t}\left(6 \sqrt{\operatorname{srank}(\Pi(S, T))}+\frac{4}{\sqrt{l}} \epsilon\right) \\
& \leq l^{2} r_{\max }^{s} r_{\max }^{t} \sqrt{\operatorname{srank}(\Pi(S, T))}(6+4 \epsilon) \leq \frac{3 w \epsilon^{2}}{\log \left(2 l / p_{\mathrm{fail}}\right)}
\end{aligned}
$$


where the equality is definition of srank, the penultimate inequality holds since $l, \operatorname{srank}(\Pi(S, T)) \geq$ 1 , and the final inequality is by (50). Substituting (52) into (49) establishes the desired result.

For Case 2, we take $M=l^{3 / 2}\|\Sigma\|_{\infty, 1} r_{\max }^{s} r_{\max }^{t}$ (Lemma G.1), and by Lemma G.2 we have

$$
m_{2}\left(X_{i}\right) \leq l\|\Sigma\|_{\infty, 1} r_{\max }^{s} r_{\max }^{t} \max \left\{\|\Pi(S, T)\|_{\infty},\|\Pi(S, T)\|_{1}\right\}
$$

We then obtain

$$
\begin{aligned}
6 \frac{m_{2}\left(X_{i}\right)}{\|\Pi(S, T)\|_{2}}+4 M \epsilon & \leq l\|\Sigma\|_{\infty, 1} r_{\max }^{s} r_{\max }^{t}\left(6 \frac{\max \left\{\|\Pi(S, T)\|_{\infty},\|\Pi(S, T)\|_{1}\right\}}{\|\Pi(S, T)\|_{2}}+4 \sqrt{l} \epsilon\right) \\
& \leq l^{3 / 2}\|\Sigma\|_{\infty, 1} r_{\max }^{s} r_{\max }^{t}(6+4 \epsilon) \leq \frac{3 w \epsilon^{2}}{\log \left(2 l / p_{\text {fail }}\right)},
\end{aligned}
$$

where the second inequality is a standard norm equivalence inequality (for $A \in \mathbb{R}^{l \times l},\|A\|_{\infty},\|A\|_{1} \leq$ $\sqrt{l}\|A\|_{2}$ ) and the third inequality is by (51). Substituting (53) into (49) completes the proof.

Lemma G.1. If $\sigma=\sigma_{\text {avg }}, \quad\left\|X_{i}\right\|_{2} \leq l^{3 / 2} r_{\max }^{s} r_{\max }^{t}$ almost surely; if $\sigma=\sigma_{\max }, \quad\left\|X_{i}\right\|_{2} \leq$ $l^{3 / 2}\|\Sigma\|_{\infty, 1} r_{\max }^{s} r_{\max }^{t}$ almost surely.

Proof. Observe $X_{i}=a_{i} b_{i}^{\top}$, where $a_{i}, b_{i} \in \mathbb{R}^{l}$ with $a_{i}(j)=r^{s_{j}}\left(\mu_{i}\right) / \sigma\left(\mu_{i}\right), b_{i}(j)=r^{t_{j}}\left(v_{i}\right) . X_{i}$ has rank 1 , and we may write its singular value decomposition as

$$
X_{i}=\left(\left\|a_{i}\right\|_{2}\left\|b_{i}\right\|_{2}\right)\left(\frac{a_{i}}{\left\|a_{i}\right\|_{2}}\right)\left(\frac{b_{i}}{\left\|b_{i}\right\|_{2}}\right)^{\top}
$$

so the nonzero singular value of $X_{i}$ is $\left\|a_{i}\right\|_{2}\left\|b_{i}\right\|_{2}$. Using the well-known fact that a matrix's 2-norm equals its largest singular value, $\left\|X_{i}\right\|_{2}=\left\|a_{i}\right\|_{2}\left\|b_{i}\right\|_{2}$, so we seek bounds on $\left\|a_{i}\right\|_{2}$ and $\left\|b_{i}\right\|_{2}$.

First, we assume $\sigma=\sigma_{\text {avg. }}$. Then we can write the following:

$$
\sigma\left(\mu_{i}\right)=\frac{1}{l} \sum_{s \in S} \frac{r^{s}\left(\mu_{i}\right)}{\left\|r^{s}\right\|_{1}} \geq \frac{1}{l r_{\max }^{s}} \sum_{s \in S} r^{s}\left(\mu_{i}\right) \geq \frac{1}{l r_{\max }^{s}}\left(\sum_{s \in S} r^{s}\left(\mu_{i}\right)^{2}\right)^{1 / 2}=\frac{1}{l r_{\max }^{s}}\left\|a_{i}\right\|_{2} \sigma\left(\mu_{i}\right) .
$$

Here, the first equality holds by definition (21), the first inequality uses the terminating condition of Algorithm $1\left(\left\|r^{s}\right\|_{1} \leq r_{\max }^{s}\right)$, the second inequality is by nonnegativity, and the second equality is by definition of $a_{i}$. We conclude $\left\|a_{i}\right\|_{2} \leq l r_{\max }^{s}$. To bound $\left\|b_{i}\right\|_{2}$, we have

$$
\left\|b_{i}\right\|_{2} \leq \sqrt{l}\left\|b_{i}\right\|_{\infty} \leq \sqrt{l} r_{\text {max }}^{t}
$$

where we have used a well-known vector norm inequality and the terminating condition of Algorithm $2\left(\left\|r^{t}\right\|_{\infty} \leq r_{\max }^{t}\right)$. Hence, $\left\|X_{i}\right\|_{2} \leq l^{3 / 2} r_{\max }^{s} r_{\max }^{t}$ follows.

Next, we assume $\sigma=\sigma_{\max }$. We have

$$
\sigma\left(\mu_{i}\right)=\frac{1}{\|\Sigma\|_{\infty, 1}} \max _{s \in S} \frac{r^{s}\left(\mu_{i}\right)}{\left\|r^{s}\right\|_{1}} \geq \frac{1}{\|\Sigma\|_{\infty, 1} r_{\max }^{s}} \max _{s \in S} r^{s}\left(\mu_{i}\right) \geq \frac{1}{l\|\Sigma\|_{\infty, 1} r_{\max }^{s}} \sum_{s \in S} r^{s}\left(\mu_{i}\right)=\frac{1}{l\|\Sigma\|_{\infty, 1} r_{\max }^{s}}\left\|a_{i}\right\| \sigma\left(\mu_{i}\right),
$$

which is justified similarly to (54). Combining with (55) gives $\left\|X_{i}\right\|_{2} \leq l^{3 / 2}\|\Sigma\|_{\infty, 1} r_{\max }^{s} r_{\max }^{t}$.

Lemma G.2. If $\sigma=\sigma_{\text {avg }}$, then $m_{2}\left(X_{i}\right) \leq l^{2} r_{\max }^{s} r_{\max }^{t}\|\Pi(S, T)\|_{F}$; if instead $\sigma=\sigma_{\max }$, then $m_{2}\left(X_{i}\right) \leq$ $l\|\Sigma\|_{\infty, 1} r_{\max }^{s} r_{\max }^{t} \max \left\{\|\Pi(S, T)\|_{\infty},\|\Pi(S, T)\|_{1}\right\}$. 


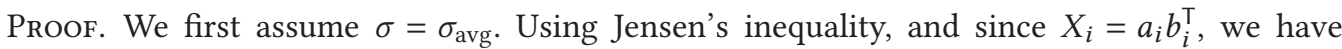
$\left\|\mathbb{E}\left[X_{i} X_{i}^{\top}\right]\right\|_{2} \leq \mathbb{E}\left[\left\|X_{i} X_{i}^{\top}\right\|_{2}\right]=\mathbb{E}\left[\left\|a_{i}\right\|_{2}^{2}\left\|b_{i}\right\|_{2}^{2}\right]$; similarly, $\left\|\mathbb{E}\left[X_{i} X_{i}^{\top}\right]\right\|_{2} \leq \mathbb{E}\left[\left\|a_{i}\right\|_{2}^{2}\left\|b_{i}\right\|_{2}^{2}\right]$. Thus,

$$
\begin{aligned}
m_{2}\left(X_{i}\right) & \leq \mathbb{E}\left[\left\|a_{i}\right\|_{2}^{2}\left\|b_{i}\right\|_{2}^{2}\right]=\sum_{u, v \in V} \sigma(u) \pi_{u}(v)\left(\frac{1}{\sigma(u)^{2}} \sum_{s \in S} r^{s}(u)^{2}\right)\left(\sum_{t \in T} r^{t}(v)^{2}\right) \\
& \leq r_{\max }^{t} \sum_{u, v \in V} \frac{\pi_{u}(v)}{\sigma(u)}\left(\sum_{s \in S} r^{s}(u)\right)^{2} \sum_{t \in T} r^{t}(v) \\
& \leq l r_{\max }^{s} r_{\max }^{t} \sum_{s \in S} \sum_{t \in T} \sum_{u, v \in V} r^{s}(u) \pi_{u}(v) r^{t}(v) \leq l r_{\max }^{s} r_{\max }^{t} \sum_{s \in S} \sum_{t \in T} \pi_{s}(t),
\end{aligned}
$$

where the second inequality uses the terminating condition of Algorithm $2\left(r^{t}(v) \leq r_{\max }^{t}\right)$ and the nonnegativity of $r^{s}(u)$, the third follows from (54), and the fourth uses the invariant (7). Finally, letting $\operatorname{vec}(\Pi(S, T))$ denote the $l^{2}$-length vector with entries $\left\{\pi_{s}(t)\right\}_{s \in S, t \in T}$, we have

$$
\sum_{s \in S} \sum_{t \in T} \pi_{S}(t)=\|\operatorname{vec}(\Pi(S, T))\|_{1} \leq l\|\operatorname{vec}(\Pi(S, T))\|_{2}=l\|\Pi(S, T)\|_{F},
$$

where the first equality is by nonnegativity, the inequality is a standard norm inequality, and the second inequality is by definition of Frobenius norm. Substituting into (57) establishes the result.

We next assume $\sigma=\sigma_{\max }$ and bound $\left\|\mathbb{E}\left[X_{i} X_{i}^{\top}\right]\right\|_{2}$. We observe that by definition,

$$
\begin{aligned}
& X_{i} X_{i}^{\top}=\frac{\left(\sum_{t \in T} r^{t}\left(v_{i}\right)^{2}\right)}{\sigma\left(\mu_{i}\right)^{2}}\left[\begin{array}{llll}
r^{s_{1}}\left(\mu_{i}\right) & \cdots & r^{s_{l}}\left(\mu_{i}\right)
\end{array}\right]^{\top}\left[\begin{array}{llll}
r^{s_{1}}\left(\mu_{i}\right) & \cdots & r^{s_{l}}\left(\mu_{i}\right)
\end{array}\right] \\
& \Rightarrow \mathbb{E}\left[X_{i} X_{i}^{\top}\right]=\sum_{u, v \in V} \frac{\pi_{u}(v)}{\sigma(u)} \sum_{t \in T} r^{t}(v)^{2}\left[\begin{array}{lllll}
r^{s_{1}}(u) & \cdots & r^{s_{l}}(u)
\end{array}\right]^{\top}\left[\begin{array}{llll}
r^{s_{1}}(u) & \cdots & r^{s_{l}}(u)
\end{array}\right] .
\end{aligned}
$$

Letting $1_{l}$ denote the all ones vector of length $l$, we also have

$$
\mathbb{E}\left[X_{i} X_{i}^{\top}\right] 1_{l}=\sum_{u, v \in V} \frac{\pi_{u}(v)}{\sigma(u)} \sum_{t \in T} r^{t}(v)^{2} \sum_{s \in S} r^{s}(u)\left[\begin{array}{llll}
r^{s_{1}}(u) & \cdots & r^{s_{l}}(u)
\end{array}\right]^{\top} .
$$

Now since $\mathbb{E}\left[X_{i} X_{i}^{\top}\right]$ is symmetric, its 2-norm is its largest eigenvalue; since it is nonnegative, the Perron-Frobenius theorem states this eigenvalue is bounded by its maximum row sum. Therefore,

$$
\begin{gathered}
\left\|\mathbb{E}\left[X_{i} X_{i}^{\mathrm{T}}\right]\right\|_{2} \leq \max _{j \in\{1,2, \ldots, l\}} \sum_{u, v \in V} \frac{\pi_{u}(v)}{\sigma(u)} \sum_{t \in T} r^{t}(v)^{2} \sum_{s \in S} r^{s}(u) r^{s_{j}}(u), \\
\leq l\|\Sigma\|_{\infty, 1} r_{\max }^{s} r_{\max }^{t} \max _{j \in\{1,2, \ldots, l\}} \sum_{t \in T} \sum_{u, v \in V} r^{s_{j}}(u) \pi_{u}(v) r^{t}(v), \\
\leq l\|\Sigma\|_{\infty, 1} r_{\max }^{s} r_{\max }^{t} \max _{j \in\{1,2, \ldots, l\}} \sum_{t \in T} \pi_{s_{j}}(t)=l\|\Sigma\|_{\infty, 1} r_{\max }^{s} r_{\max }^{t}\|\Pi(S, T)\|_{\infty},
\end{gathered}
$$

where (59) uses the row sums derived in (58), (60) uses (56) from the proof of Lemma G.1 and the terminating condition of Algorithm $2\left(\left\|r^{t}\right\|_{\infty} \leq r_{\max }^{t}\right)$, and (61) uses the invariant (7). We can use the same idea to bound $\left\|\mathbb{E}\left[X_{i}^{\top} X_{i}\right]\right\|_{2}$. The steps to obtain the expression analogous to (59) follow 
the same approach, so we omit them. We then have

$$
\begin{aligned}
&\left\|\mathbb{E}\left[X_{i}^{\top} X_{i}\right]\right\|_{2} \leq \max _{j \in\{1,2, \ldots, l\}} \sum_{u, v \in V} \frac{\pi_{u}(v)}{\sigma(u)} \sum_{s \in S} r^{s}(u)^{2} \sum_{t \in T} r^{t}(v) r^{t_{j}}(v) \\
& \leq \sum_{u, v \in V} \frac{\pi_{u}(v)}{\sigma(u)} \sum_{s \in S} r^{s}(u) \max _{s^{\prime} \in S} r^{s^{\prime}}(u) \sum_{t \in T} r^{t}(v) r^{t_{j}}(v), \\
& \leq l\|\Sigma\|_{\infty, 1} r_{\max }^{s} r_{\max }^{t} \max _{j \in\{1,2, \ldots, l\}} \sum_{s \in S} \sum_{u, v \in V} r^{s}(u) \pi_{u}(v) r^{t_{j}}(v), \\
& \leq l\|\Sigma\|_{\infty, 1} r_{\max }^{s} r_{\max }^{t} \max _{j \in\{1,2, \ldots, l\}} \sum_{s \in S} \pi_{s}\left(t_{j}\right)=l\|\Sigma\|_{\infty, 1} r_{\max }^{s} r_{\max }^{t}\|\Pi(S, T)\|_{1},
\end{aligned}
$$

where (62) is immediate, (63) uses (56) from the proof of Lemma G.1 and the terminating condition of Algorithm $2\left(\left\|r^{t}\right\|_{\infty} \leq r_{\max }^{t}\right)$, and (64) uses the invariant (7). We conclude from (61) and (64) that

$$
\max \left\{\left\|\mathbb{E}\left[X_{i}^{\top} X_{i}\right]\right\|_{2},\left\|\mathbb{E}\left[X_{i} X_{i}^{\top}\right]\right\|_{2}\right\} \leq l\|\Sigma\|_{\infty, 1} r_{\max }^{s} r_{\max }^{t} \max \left\{\|\Pi(S, T)\|_{\infty},\|\Pi(S, T)\|_{1}\right\} .
$$

\section{REFERENCES}

[1] Reid Andersen, Christian Borgs, Jennifer Chayes, John Hopcroft, Vahab Mirrokni, and Shang-Hua Teng. 2008. Local computation of PageRank contributions. Internet Mathematics 5, 1-2 (2008), 23-45.

[2] Reid Andersen, Fan Chung, and Kevin Lang. 2006. Local graph partitioning using PageRank vectors. In Proceedings of the 2006 47th Annual IEEE Symposium on Foundations of Computer Science (FOCS’06). IEEE, Los Alamitos, CA, 475-486.

[3] David Arthur and Sergei Vassilvitskii. 2007. $k$-means++: The advantages of careful seeding. In Proceedings of the 18th Annual ACM-SIAM Symposium on Discrete Algorithms. 1027-1035.

[4] Krishna B. Athreya and Örjan Stenflo. 2003. Perfect sampling for Doeblin chains. Sankhyā: The Indian fournal of Statistics 65, 4 (2003), 763-777.

[5] Konstantin Avrachenkov, Nelly Litvak, Danil Nemirovsky, and Natalia Osipova. 2007. Monte Carlo methods in PageRank computation: When one iteration is sufficient. SIAM fournal on Numerical Analysis 45, 2 (2007), 890-904.

[6] Shumeet Baluja, Rohan Seth, D. Sivakumar, Yushi Jing, Jay Yagnik, Shankar Kumar, Deepak Ravichandran, and Mohamed Aly. 2008. Video suggestion and discovery for YouTube: Taking random walks through the view graph. In Proceedings of the 17th International Conference on World Wide Web. ACM, New York, NY, 895-904.

[7] Christian Borgs, Michael Brautbar, Jennifer Chayes, and Shang-Hua Teng. 2014. Multiscale matrix sampling and sublinear-time PageRank computation. Internet Mathematics 10, 1-2 (2014), 20-48.

[8] Devdatt P. Dubhashi and Alessandro Panconesi. 2009. Concentration of Measure for the Analysis of Randomized Algorithms. Cambridge University Press.

[9] Dániel Fogaras, Balázs Rácz, Károly Csalogány, and Tamás Sarlós. 2005. Towards scaling fully personalized PageRank: Algorithms, lower bounds, and experiments. Internet Mathematics 2, 3 (2005), 333-358.

[10] Valerio Freschi. 2007. Protein function prediction from interaction networks using a random walk ranking algorithm. In Proceedings of the 7th IEEE International Conference on Bioinformatics and Bioengineering. IEEE, Los Alamitos, CA, $42-48$.

[11] David F. Gleich. 2015. PageRank beyond the Web. SIAM Review 57, 3 (2015), 321-363.

[12] Pankaj Gupta, Ashish Goel, Jimmy Lin, Aneesh Sharma, Dong Wang, and Reza Zadeh. 2013. WTF: The who to follow service at Twitter. In Proceedings of the 22nd International Conference on World Wide Web. ACM, New York, NY, 505-514.

[13] Taher H. Haveliwala. 2002. Topic-sensitive PageRank. In Proceedings of the 11th International Conference on World Wide Web. ACM, New York, NY, 517-526.

[14] Glen Jeh and Jennifer Widom. 2003. Scaling personalized Web search. In Proceedings of the 12th International Conference on World Wide Web. ACM, New York, NY, 271-279.

[15] Danai Koutra, Joshua T. Vogelstein, and Christos Faloutsos. 2013. Deltacon: A principled massive-graph similarity function. In Proceedings of the 2013 SIAM International Conference on Data Mining. 162-170.

[16] Christina E. Lee, Asuman Ozdaglar, and Devavrat Shah. 2013. Computing the stationary distribution locally. In Advances in Neural Information Processing Systems. 1376-1384.

[17] Christina E. Lee, Asuman Ozdaglar, and Devavrat Shah. 2014. Asynchronous approximation of a single component of the solution to a linear system. arXiv:1411.2647. 
[18] Christina E. Lee, Asuman E. Ozdaglar, and Devavrat Shah. 2014. Solving systems of linear equations: Locally and asynchronously. arXiv:1411.2647v1.

[19] Jure Leskovec and Andrej Krevl. [n.d.]. Stanford Large Network Dataset Collection. Retrieved November 11, 2019 from http://snap.stanford.edu/data.

[20] Peter Lofgren, Siddhartha Banerjee, and Ashish Goel. 2016. Personalized PageRank estimation and search: A bidirectional approach. In Proceedings of the 9th ACM International Conference on Web Search and Data Mining. ACM, New York, NY, 163-172.

[21] Peter Lofgren and Ashish Goel. 2013. Personalized PageRank to a target node. arXiv:1304.4658.

[22] Sean P. Meyn and Richard L. Tweedie. 2012. Markov Chains and Stochastic Stability. Springer Science \& Business Media.

[23] Julie L. Morrison, Rainer Breitling, Desmond J. Higham, and David R. Gilbert. 2005. GeneRank: Using search engine technology for the analysis of microarray experiments. BMC Bioinformatics 6, 1 (2005), 233.

[24] Lawrence Page, Sergey Brin, Rajeev Motwani, and Terry Winograd. 1999. The PageRank Citation Ranking: Bringing Order to the Web. Technical Report. Stanford InfoLab.

[25] James Gary Propp and David Bruce Wilson. 1996. Exact sampling with coupled Markov chains and applications to statistical mechanics. Random Structures and Algorithms 9, 1-2 (1996), 223-252.

[26] Kijung Shin, Jinhong Jung, Sael Lee, and U. Kang. 2015. Bear: Block elimination approach for random walk with restart on large graphs. In Proceedings of the 2015 ACM SIGMOD International Conference on Management of Data. ACM, New York, NY, 1571-1585.

[27] Hanghang Tong, Christos Faloutsos, and Jia-Yu Pan. 2008. Random walk with restart: Fast solutions and applications. Knowledge and Information Systems 14, 3 (2008), 327-346.

[28] Joel A. Tropp. 2015. An introduction to matrix concentration inequalities. Foundations and Trends in Machine Learning 8, 1-2 (2015), 1-230.

[29] Daniel Vial and Vijay Subramanian. 2018. On the role of clustering in Personalized PageRank estimation. arXiv:1706.01091.

Received July 2018; revised July 2019; accepted October 2019 